\title{
Structure of inverse limit spaces of tent maps with finite critical orbit
}

\author{
by
}

Sonja Štimac (Zagreb)

\begin{abstract}
Using methods of symbolic dynamics, we analyze the structure of composants of the inverse limit spaces of tent maps with finite critical orbit. We define certain symmetric arcs called bridges. They are building blocks of composants. Then we show that the folding patterns of bridges are characterized by bridge types and prove that there are finitely many bridge types.
\end{abstract}

1. Introduction. The one-parameter family of tent maps on the unit interval is an important family of one-dimensional maps, because it exemplifies a variety of dynamical phenomena encountered in more general families of one-dimensional maps. The observation that inverse limit spaces of onedimensional maps appear as attractors in dynamical systems has generated considerable interest in such spaces. The inverse limit spaces, formed by using a single tent map for all the bonding maps, provide a one-parameter family of models for Hénon and other generalized horseshoe attractors. Various authors have been interested in the topology of such inverse limit spaces with an eye to a further understanding of these attractors (for instance, C. Bandt in [B], M. Barge, K. M. Brucks and B. Diamond in [Ba-Br-D], M. Barge and W. T. Ingram in [Ba-I], H. Bruin in [Brn1] and [Brn3], W. T. Ingram in [I] and L. Kailhofer in [K1] and [K2]).

If inverse limit spaces are to be used to classify dynamical systems, then it is of fundamental importance to be able to determine whether or not two inverse limit spaces are homeomorphic. Therefore, the understanding of the structure of such inverse limit spaces is also an interesting and important task. In 1992, W. T. Ingram conjectured that the inverse limit spaces based on two tent maps with different slopes are not homeomorphic. This conjec-

2000 Mathematics Subject Classification: 37B10, 37B45.

Key words and phrases: tent map, inverse limit, composant, folding point, folding pattern.

Supported in part by the MZT Grant 0037105 of the Republic of Croatia. 
ture has recently received significant attention. Of particular interest is the simplest case when the tent maps have finite critical orbits. Several authors have addressed the difficult question of determining when such inverse limit spaces are homeomorphic (for example, M. Barge and B. Diamond in [Ba-D], H. Bruin in [Brn2], L. Kailhofer in [K1] and [K2], and S. Štimac in [ک̌̉]).

The purpose of this paper is to develop a method for describing the structure of the inverse limit spaces of a certain family of tent maps. In a forthcoming paper this method will be used in the classification of inverse limit spaces of tent maps with finite critical orbits.

For $s \in(1,2]$, let $T_{s}:[0,1] \rightarrow[0,1]$ be the tent map with slope $s$, i.e.

$$
T_{s}(\xi)= \begin{cases}s \xi & \text { if } 0 \leq \xi \leq 1 / 2, \\ s(1-\xi) & \text { if } 1 / 2 \leq \xi \leq 1 .\end{cases}
$$

Let $K_{s}$ denote the limit of the inverse sequence consisting of copies of $[0,1]$ and tent maps $T_{s}$,

$$
\begin{aligned}
K_{s} & =\lim _{\longleftarrow}\left\{[0,1], T_{s}\right\} \\
& =\left\{\left(\ldots, \xi_{-3}, \xi_{-2}, \xi_{-1}\right) \in[0,1]^{\mathbb{N}}: \xi_{-i}=T_{s}\left(\xi_{-i-1}\right), i \in \mathbb{N}\right\} .
\end{aligned}
$$

Although the notation for the points of $K_{s}$ may seem somewhat unusual, it turned out to have practical advantages in our case. $K_{s}$ is a continuum (compact connected metric space) which is indecomposable [I]. Since $K_{2}$ is known by the name of Knaster continuum (or bucket handle continuum), we will call $K_{s}$ the generalized Knaster continua.

Similarly to [Ba-I], one can prove that for $s \in(\sqrt{2}, 2]$, the continuum $K_{s}$ is the union of the continuum $\lim \left\{J_{s},\left.T_{s}\right|_{J_{s}}\right\}$ and of the half-line $R$ entwined in it, so that cl $R \backslash R=\lim \left\{\left.\overleftarrow{J_{s}, T_{s}}\right|_{J_{s}}\right\}$, where $J_{s}=\left[T_{s}^{2}(1 / 2), T_{s}(1 / 2)\right]$ and $\left.T_{s}\right|_{J_{s}}$ is the core of the tent map $T_{s}$.

For $s \in(1, \sqrt{2}]$, the continuum $K_{s}$ is the union of a half-line $R$ and of two continua $C^{1}$ and $C^{2}$ such that $\operatorname{cl} R \backslash R=C^{1} \cup C^{2}, C^{1} \cap C^{2}$ is a point, and $C^{1}$ and $C^{2}$ are homeomorphic to the continuum $K_{s^{2}}$. Therefore, to describe the structure of the continua $K_{s}, s \in(1,2]$, it is sufficient to describe the structure of the continua $\lim _{\longleftarrow}\left\{J_{s},\left.T_{s}\right|_{J_{s}}\right\}, s \in(\sqrt{2}, 2]$, or analogously, the continua from the family $C_{s}=\lim \left\{[0,1], f_{s}\right\}, s \in(\sqrt{2}, 2]$, where $f_{s}:[0,1] \rightarrow$ $[0,1]$ are the rescaled cores of the tent maps $T_{s}$,

$$
f_{s}(\xi)= \begin{cases}s \xi+2-s & \text { if } 0 \leq \xi \leq c_{s} \\ s(1-\xi) & \text { if } c_{s} \leq \xi \leq 1\end{cases}
$$

with $c_{s}=(s-1) / s$.

A point $x \in C_{s}$ is called an endpoint of $C_{s}$ if for every pair of subcontinua $A, B$ of $C_{s}$ with $x \in A \cap B$, either $A \subseteq B$ or $B \subseteq A$. The continuum $C_{s}$ has $N \in \mathbb{N}$ endpoints if and only if 0 is a periodic point of $f_{s}$ with period $N$. The continuum $C_{s}$ has infinitely many endpoints if and only if 0 is a recurrent 
but not periodic point of $f_{s}$. Finally, $C_{s}$ does not have endpoints if and only if 0 is not a recurrent point of $f_{s}$. These results were proved in [Ba-M].

It is well known that the continua $C_{s}$ are chainable. A chain is a finite open cover $\mathcal{C}=\left\{L_{i}\right\}_{i=1}^{n}$ of $C_{s}$ whose links $L_{i}$ and $L_{j}$ intersect if and only if $|i-j| \leq 1$. A space is said to be chainable if for every $\epsilon>0$ there is a chain whose links have diameter less than $\epsilon$. If $\mathcal{C}$ and $\mathcal{C}^{\prime}$ are chains, $\mathcal{C}$ is called finer than $\mathcal{C}^{\prime}$ if for every link $L \in \mathcal{C}$ there is a link $L^{\prime} \in \mathcal{C}^{\prime}$ containing $L$. A link $L \in \mathcal{C}$ is a turnlink if there exist an adjacent link $M \in \mathcal{C}$, a chain $\mathcal{C}^{\prime}=\left\{L_{i}^{\prime}\right\}_{i=1}^{n^{\prime}}$ and integers $a, b, 1 \leq a<b \leq n^{\prime}$, such that $\bigcup_{i=a}^{b} L_{i}^{\prime} \subset L \cup M$, $\left(\bigcup_{i=a}^{b} L_{i}^{\prime}\right) \cap L \neq \emptyset$ and $L_{a}^{\prime}, L_{b}^{\prime} \subset M \backslash L$. In this case we say that $\mathcal{C}^{\prime}$ turns in $L$. The link $L$ is an essential turnlink if every sufficiently fine chain $\mathcal{C}^{\prime}$ has a turnlink in $L$. A point $x \in C_{s}$ is a folding point if for every neighborhood $U$ of $x$, every sufficiently fine chain has a turnlink (and therefore, an essential turnlink) in $U$.

A folding point $x$ can be either one-sided or two-sided. Assume that $\mathcal{C}$ is a chain and a link $L$ containing $x$ is neither the first nor the last link. Then $x$ is one-sided if there is a single link $M$, adjacent to $L$, such that every sufficiently fine chain turns in $L \cup M$. If $M^{\prime}$ is the other adjacent link and sufficiently fine chains turn both in $L \cup M$ and $L \cup M^{\prime}$, then $x$ is a two-sided folding point. An example of a one-sided folding point is the endpoint of the bucket handle $C_{2}$. A nice illustration of a two-sided folding point appears in the inverse limit space $C_{\sqrt{2}}$ of the tent map with slope $\sqrt{2}$. In this case $f_{\sqrt{2}}(0)$ is the fixed point and $C_{\sqrt{2}}$ consists of two bucket handles glued together at their endpoints. The glue point is the unique two-sided folding point [Ba-I].

If $c_{s}$ is a periodic point of $f_{s}$ with period $N$, i.e. $f_{s}^{N}\left(c_{s}\right)=c_{s}$ and $f_{s}^{i}\left(c_{s}\right) \neq c_{s}$ for $0<i<N$, the continuum $C_{s}$ has $N$ endpoints and these points are the only folding points of $C_{s}$. Every endpoint is a one-sided folding point. If $c_{s}$ is a strictly preperiodic point of $f_{s}$, i.e. $f_{s}^{M}\left(c_{s}\right)=\xi=f_{s}^{N}(\xi)$, $M \neq 0$ and $f_{s}^{i}(\xi) \neq \xi$, for $0<i<N$, then the continuum $C_{s}$ has $N$ folding points which are not endpoints, with the exception of the bucket handle $C_{2}$ whose only folding point is an endpoint. If $\xi$ is orientation-preserving, then the corresponding folding point is one-sided. If $\xi$ is orientation-reversing, the corresponding folding point is two-sided. With the exception of the folding points, the inverse limit space of a tent map with periodic or strictly preperiodic critical point is locally homeomorphic to a Cantor set of arcs [Brn2].

From now on, we will consider continua $C_{s}, s \in(\sqrt{2}, 2]$, such that the corresponding bonding maps $f_{s}$ have finite critical orbits.

In Section 2 we use C. Bandt's [B] and K. M. Bruck and B. Diamond's [Br-D] ideas of representing $C_{s}$ as the quotient space of the space of two-sided 
allowed sequences of two symbols 0,1 with respect to a certain equivalence relation $\approx$. Then we give properties of the two-sided allowed sequences and we define an ordering $\preceq$ on every composant. We also define some special points of $C_{s}$, called $i$-points, and we define and analyze certain arcs in the composants of $C_{s}$, called basic arcs.

In Section 3 we first describe in detail the properties of the folding patterns of composants with one folding point. Then we define certain symmetric arcs in the composants, called $p$-bridges. Analyzing them we show that their folding patterns are characterized by a number called the bridge type. We prove (Theorem 3.20) that there are finitely many bridge types. Using these folding patterns, we discuss the folding pattern of any composant having no folding points. L. Kailhofer gave in [K1] and [K2] many properties of these patterns of the composant of a particular endpoint of a continuum $C_{s}$ with finitely many endpoints. In distinction to the topological methods used by L. Kailhofer, we systematically apply the methods of symbolic dynamics and coding.

2. Coding generalized Knaster continua. Let $M \in \mathbb{Z}_{+}, N \in \mathbb{N}$ and $s \in(\sqrt{2}, 2]$ be such that $c_{s}$ is preperiodic under $f_{s}$ with preperiod $M$ and period $N$, i.e., there is $\xi \in[0,1]$ with $f_{s}^{M}\left(c_{s}\right)=\xi, f_{s}^{N}(\xi)=\xi$ and $f_{s}^{i}(\xi) \neq \xi$ for $0<i<N$. This means that the orbit of the point $c_{s}, \mathcal{O}_{s}\left(c_{s}\right)=$ $\left\{c_{s}, f_{s}\left(c_{s}\right)=1, f_{s}^{2}\left(c_{s}\right)=0, f_{s}^{3}\left(c_{s}\right), \ldots\right\}$, has $M+N$ points. Note that when $M=0$, we are in a periodic case, and when $M>2$, we are in a strictly preperiodic case. Let $\mathcal{O}_{s}\left(c_{s}\right)=\left\{0=\xi_{0}<\xi_{1}<\cdots<\xi_{M+N-1}=1\right\}$, and let $I_{i}=\left[\xi_{i}, \xi_{i+1}\right], i \in\{0, \ldots, M+N-2\}$. The family of closed subintervals $\left\{I_{i}\right\}$ of the interval $[0,1]$ forms a partition since the interiors of the intervals $I_{i}$ are pairwise disjoint. Note that $\bigcup_{i=0}^{M+N-2} I_{i}=[0,1]$. The map $f_{s}$ is a Markov map, i.e., it is surjective, $\mathcal{C}^{1}$ and monotone on each of the open intervals $\operatorname{int} I_{i}$, and has the following properties:

(1) there exists $\alpha>1$ such that $\left|f_{s}^{\prime}(x)\right| \geq \alpha$ for each $x \in \operatorname{int} I_{i}, i \in$ $\{0, \ldots, M+N-2\}$

(2) if $f_{s}\left(\right.$ int $\left.I_{i}\right) \cap$ int $I_{j} \neq \emptyset$, then $f_{s}\left(\right.$ int $\left.I_{i}\right) \supseteq \operatorname{int} I_{j}$ for $i, j \in\{0, \ldots, M+$ $N-2\}$

([P-Y, p. 39]). The map $f_{s}$ is also locally eventually onto (l.e.o.), i.e., for every interval $J \subset[0,1]$ there exists $n \in \mathbb{N}$ with $f_{s}^{n}(J)=[0,1]([\mathrm{P}-\mathrm{Y}, \mathrm{p} .40])$. The Markov graph of $f_{s}$ associated with the partition $\left\{I_{i}\right\}$ is the graph whose vertices are the intervals of the partition and the edges are the pairs $\left(I_{j}, I_{k}\right)$ such that $f_{s}\left(I_{j}\right) \supseteq I_{k}$. Such an edge is denoted by $I_{j} \rightarrow I_{k}$ ([M-S, p. 83]). Note that either $I_{i} \subseteq I^{0}$ or $I_{i} \subseteq I^{1}$ with $I^{0}=\left[0, c_{s}\right]$ and $I^{1}=\left[c_{s}, 1\right]$.

Let $I_{i_{0}} \rightarrow I_{i_{1}} \rightarrow \cdots \rightarrow I_{i_{k}} \rightarrow \cdots$ be a path in the Markov graph (finite or infinite). To every such path we assign the sequence $x_{0} x_{1} \ldots x_{k} \ldots$ (finite 
or infinite) defined by

$$
x_{j}= \begin{cases}0, & I_{i_{j}} \subseteq I^{0} \\ 1, & I_{i_{j}} \subseteq I^{1}\end{cases}
$$

We then say that the path and the sequence are associated. A finite or infinite sequence of zeros and ones is called allowed (with respect to $f_{s}$ ) if it is associated with some path in the Markov graph. If a sequence $x_{0} x_{1} \ldots x_{k} \ldots$ is allowed, then all of its finite parts $x_{j} \ldots x_{j+k}$ are allowed.

LEMMA 2.1. Let $x_{0} x_{1} \ldots x_{n+M+N-1}, n \in \mathbb{Z}_{+}$, be an allowed sequence of length $n+M+N$. Then there exist at most two different paths of length $n+1$ in the Markov graph of $f_{s}$ with the following property: Any path $I_{i_{0}} \rightarrow$ $I_{i_{1}} \rightarrow \cdots \rightarrow I_{i_{n+M+N-1}}$ in the Markov graph of $f_{s}$ associated with the given sequence starts with one of these two paths of length $n+1$. Moreover, if $M=0$ and $n=0$, then every path in the Markov graph associated with the sequence $x_{0} x_{1} \ldots x_{N-1}$ starts with the same vertex.

Proof. Let $I_{i_{0}} \rightarrow I_{i_{1}} \rightarrow \cdots \rightarrow I_{i_{n+M+N-1}}, I_{j_{0}} \rightarrow I_{j_{1}} \rightarrow \cdots \rightarrow I_{j_{n+M+N-1}}$ and $I_{l_{0}} \rightarrow I_{l_{1}} \rightarrow \cdots \rightarrow I_{l_{n+M+N-1}}$ be three paths in the Markov graph, all associated with $x_{0} x_{1} \ldots x_{n+M+N-1}$. Let $J_{k}=\operatorname{conv}\left(I_{i_{k}} \cup I_{j_{k}} \cup I_{l_{k}}\right), k \in$ $\{0, \ldots, n+M+N-1\}$, where $\operatorname{conv}(A)$ denotes the convex hull of $A$. Since the same element $x_{k}$ of the allowed sequence $x_{0} x_{1} \ldots x_{n+M+N-1}$ is associated with the vertices $I_{i_{k}}, I_{j_{k}}$ and $I_{l_{k}}$, the intervals $I_{i_{k}}, I_{j_{k}}$ and $I_{l_{k}}$ all lie either to the left of $c_{s}$, or to the right of $c_{s}$. So, $c_{s} \notin$ int $J_{k}$ and $\left.f_{s}\right|_{J_{k}}$ is strictly monotone, for every $k \in\{0, \ldots, n+M+N-1\}$. Because of that, and since $f_{s}\left(I_{i_{k}}\right) \supseteq I_{i_{k+1}}, f_{s}\left(I_{j_{k}}\right) \supseteq I_{j_{k+1}}$ and $f_{s}\left(I_{l_{k}}\right) \supseteq I_{l_{k+1}}$, we conclude that $I_{i_{k}} \neq I_{j_{k}} \neq I_{l_{k}} \neq I_{i_{k}}$ implies $I_{i_{k+1}} \neq I_{j_{k+1}} \neq I_{l_{k+1}} \neq I_{i_{k+1}}$.

Suppose that $I_{i_{n}} \neq I_{j_{n}} \neq I_{l_{n}} \neq I_{i_{n}}$. Then $I_{i_{k}} \neq I_{j_{k}} \neq I_{l_{k}} \neq I_{i_{k}}$ for every $k \in\{n, \ldots, n+M+N-1\}$. Without loss of generality we can assume that $i_{n}<j_{n}<l_{n}$. Let $M \neq 0$ and $I_{j_{k}}=\left[\xi_{j_{k}}, \xi_{j_{k+1}}\right]$ for every $k \in\{n, \ldots$, $n+M+N-1\}$. Then $I_{j_{k}} \subset$ int $J_{k}$ for every $k \in\{n, \ldots, n+M+N-1\}$. Since $\xi_{j_{n}}, \xi_{j_{n+1}} \in \mathcal{O}\left(c_{s}\right)$, there is $m \in \mathbb{N}, m<M$, such that $f_{s}^{m}\left(\xi_{j_{n}}\right)=$ $\xi_{a}=f_{s}^{N}\left(\xi_{a}\right) \in \mathcal{O}\left(c_{s}\right)$ and $f_{s}^{m}\left(\xi_{j_{n+1}}\right)=\xi_{b}=f_{s}^{N}\left(\xi_{b}\right) \in \mathcal{O}\left(c_{s}\right)$. Without loss of generality we can assume that $\xi_{a}<\xi_{b}$. Since $\left.f_{s}^{k}\right|_{\left[\xi_{a}, \xi_{b}\right]}$ is strictly monotone for every $k \in\{0, \ldots, N\}$, it follows that $f_{s}^{N}\left(\left[\xi_{a}, \xi_{b}\right]\right)=\left[\xi_{a}, \xi_{b}\right]$, which contradicts $f_{s}$ being l.e.o. Hence, $I_{i_{k}}=I_{j_{k}}$ for every $k \in\{0, \ldots, n\}$, or $I_{j_{k}}=I_{l_{k}}$ for every $k \in\{0, \ldots, n\}$.

Let $M=0, n=0$ and $I_{l_{k}}=\emptyset$ for every $k \in\{0, \ldots, N-1\}$. Then $f_{s}^{k}\left(\xi_{i_{0}+1}\right) \in \operatorname{int} J_{k}$ for every $k \in\{0, \ldots, N-1\}$. Since $\xi_{i_{0}+1} \in \mathcal{O}\left(c_{s}\right)$, there is $K \in \mathbb{N}, K \leq N-1$, such that $f_{s}^{K}\left(\xi_{i_{0}+1}\right)=c_{s}$, contrary to $c_{s} \notin$ int $J_{k}$ for every $k \in\{0, \ldots, N-1\}$. Hence, $I_{i_{0}}=I_{j_{0}}$.

Let us consider an example in which, for an allowed sequence of length $n+M+N$, there are two different paths of length $n+1$ with the property 
that any associated path in the Markov graph starts with one of these two paths of length $n+1$. Let us denote $f_{s}^{k}\left(c_{s}\right)$ by $c^{k}$ for every $k \in \mathbb{N}$. Then $c^{1}=1$ and $c^{2}=0$.

EXAMPLE 2.2. Let $s \in[\sqrt{2}, 2]$ be such that the mapping $f_{s}$ is strictly preperiodic with $M=3, N=2, c^{3}<c_{s}$ and $c^{4}>c_{s}$. It is easy to see that such an $s$ exists $(s=1.69562 \ldots)$. Then $\mathcal{O}\left(c_{s}\right)=\left\{0<c^{3}<c_{s}<c^{4}<1\right\}$ and $I_{0}=\left[0, c^{3}\right], I_{1}=\left[c^{3}, c_{s}\right], I_{2}=\left[c_{s}, c^{4}\right]$ and $I_{3}=\left[c^{4}, 1\right]$. The Markov graph of $f_{s}$ looks like

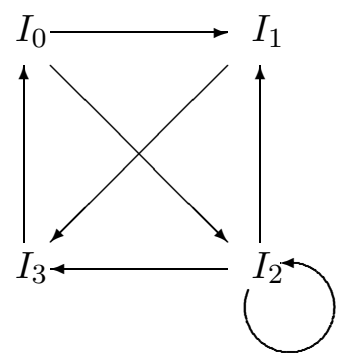

For the sequence 01010101 of length 8 , let $I_{i_{0}} \rightarrow \cdots \rightarrow I_{i_{7}}$ be an arbitrary associated path in the Markov graph. Then the initial part of length 3 can be either $I_{1} \rightarrow I_{3} \rightarrow I_{0}$, or $I_{0} \rightarrow I_{2} \rightarrow I_{1}$.

Proposition 2.3. A sequence $x_{0} x_{1} x_{2} \ldots \in\{0,1\}^{\mathbb{Z}_{+}}$is allowed if and only if, for every $k \in \mathbb{N}, k \geq M+N$, the initial part $x_{0} x_{1} \ldots x_{k}$ of length $k+1$ is allowed.

Proof. Let $x_{0} x_{1} \ldots \in\{0,1\}^{\mathbb{Z}_{+}}$be such that, for every $k \in \mathbb{N}, k \geq M+N$, the finite sequence $x_{0} \ldots x_{k}$ is allowed. Therefore, for any $n \in \mathbb{Z}_{+}$, the finite sequence $x_{0} \ldots x_{n+M+N-1}$ is allowed. By Lemma 2.1, at most two different initial parts of length $n+1$ can start the associated paths in the Markov graph. Denote them by $I_{i_{0}} \rightarrow I_{i_{1}} \rightarrow \cdots \rightarrow I_{i_{n}}$ and $I_{j_{0}} \rightarrow I_{j_{1}} \rightarrow \cdots \rightarrow I_{j_{n}}$. We want to prove that at least one of these finite paths can be extended to a path in the Markov graph associated with $x_{0} x_{1} \ldots$

Since the finite sequence $x_{0} \ldots x_{n+M+N}$ is allowed, by Lemma 2.1 there are at most two different initial parts of length $n+2$. Denote them by $I_{l_{0}} \rightarrow I_{l_{1}} \cdots \rightarrow I_{l_{n+1}}$ and $I_{k_{0}} \rightarrow I_{k_{1}} \cdots \rightarrow I_{k_{n+1}}$. If the paths $I_{l_{0}} \rightarrow \cdots \rightarrow I_{l_{n}}$ and $I_{k_{0}} \rightarrow \cdots \rightarrow I_{k_{n}}$ are different, then either $I_{l_{0}} \rightarrow \cdots \rightarrow I_{l_{n}}$ is the same as $I_{i_{0}} \rightarrow \cdots \rightarrow I_{i_{n}}$ and $I_{k_{0}} \rightarrow \cdots \rightarrow I_{k_{n}}$ is the same as $I_{j_{0}} \rightarrow \cdots \rightarrow I_{j_{n}}$, or $I_{l_{0}} \rightarrow \cdots \rightarrow I_{l_{n}}$ is the same as $I_{j_{0}} \rightarrow \cdots \rightarrow I_{j_{n}}$ and $I_{k_{0}} \rightarrow \cdots \rightarrow I_{k_{n}}$ is the same as $I_{i_{0}} \rightarrow \cdots \rightarrow I_{i_{n}}$. In that case we can extend both paths.

If $I_{l_{0}} \rightarrow \cdots \rightarrow I_{l_{n}}$ is the same as $I_{k_{0}} \rightarrow \cdots \rightarrow I_{k_{n}}$ then we cannot extend both paths. Without loss of generality we can assume that $I_{i_{0}} \rightarrow \cdots \rightarrow I_{i_{n}}$ is the same as $I_{l_{0}} \rightarrow \cdots \rightarrow I_{l_{n}}$. So, the path which cannot be extended is $I_{j_{0}} \rightarrow \cdots \rightarrow I_{j_{n}}$. In that case we should prove that for every finite sequence $x_{0} \ldots x_{k+M+N}, k>n$, every associated path in the Markov graph starts 
with $I_{i_{0}} \rightarrow \cdots \rightarrow I_{i_{n}}$. By Lemma 2.1, there are at most two different initial parts of length $k+1$. If for one of them, $I_{m_{0}} \rightarrow \cdots \rightarrow I_{m_{k}}$, the paths $I_{m_{0}} \rightarrow \cdots \rightarrow I_{m_{n}}$ and $I_{i_{0}} \rightarrow \cdots \rightarrow I_{i_{n}}$ are different, then $I_{m_{0}} \rightarrow \cdots \rightarrow I_{m_{n}}$ and $I_{j_{0}} \rightarrow \cdots \rightarrow I_{j_{n}}$ are the same. Therefore the path $I_{j_{0}} \rightarrow \cdots \rightarrow I_{j_{n}}$ can be extended as $I_{j_{0}} \rightarrow \cdots \rightarrow I_{j_{n}} \rightarrow I_{m_{n+1}}$, contrary to the assumption that $I_{l_{0}} \rightarrow \cdots \rightarrow I_{l_{n}}$ and $I_{k_{0}} \rightarrow \cdots \rightarrow I_{k_{n}}$ are the same.

The argument for the opposite implication is trivial.

LEMmA 2.4. Let $M=0$ and let $x_{0} x_{1} \ldots x_{N+1}$ be a sequence of length $N+2$. If $x_{0} \ldots x_{N}$ and $x_{1} \ldots x_{N+1}$ are allowed sequences, then the sequence $x_{0} \ldots x_{N+1}$ is also allowed.

Proof. Let $I_{i_{0}} \rightarrow I_{i_{1}} \rightarrow \cdots \rightarrow I_{i_{N}}$ be a path in the Markov graph associated with $x_{0} x_{1} \ldots x_{N}$ and let $I_{j_{1}} \rightarrow I_{j_{2}} \rightarrow \cdots \rightarrow I_{j_{N+1}}$ be a path associated with $x_{1} \ldots x_{N+1}$. Since the sequence $x_{1} \ldots x_{N}$ is allowed and it is of length $N$, by Lemma 2.1 every path associated with it starts with the same vertex. So, $I_{i_{1}}=I_{j_{1}}$ and there is a path $I_{i_{0}} \rightarrow I_{j_{1}} \rightarrow I_{j_{2}} \rightarrow \cdots \rightarrow I_{j_{N+1}}$. Since the sequence $x_{0} x_{1} \ldots x_{N+1}$ is associated with the path $I_{i_{0}} \rightarrow I_{j_{1}} \rightarrow$ $I_{j_{2}} \rightarrow \cdots \rightarrow I_{j_{N+1}}$, this sequence is allowed.

There is no analogous statement for the strictly preperiodic case. Let us consider the Markov graph from Example 2.1. It is easy to see that for every $n \in \mathbb{N}$, the sequences $0(01)^{n}$ and $(01)^{n} 00$ are allowed. But the sequence $0(01)^{2 n} 00$ is not allowed, for every $n \in \mathbb{N}$. Here, for a finite sequence $x_{0} \ldots x_{k}$, we write $\left(x_{0} \ldots x_{k}\right)^{n}=\underbrace{x_{0} \ldots x_{k}}_{n \text { times }} \underbrace{x_{0} \ldots x_{k}} \cdots \underbrace{x_{0} \ldots x_{k}}$.

Proposition 2.5. Let $M=0$. A sequence $x_{0} x_{1} \ldots \in\{0,1\}^{\mathbb{Z}_{+}}$is allowed if and only if all of its finite parts of length $N+1$ are allowed, i.e., if and only if, for every $j \in \mathbb{Z}_{+}$, the finite sequence $x_{j} x_{j+1} \ldots x_{j+N}$ is allowed.

Proof. This follows by Proposition 2.3 and Lemma 2.4.

Since we will work with several types of sequences, to avoid confusion, we denote:

- left-infinite sequences by $\overleftarrow{x}=\left(x_{-i}\right)_{i \in \mathbb{N}}=\ldots x_{-3} x_{-2} x_{-1}$,

- right-infinite sequences by $\vec{x}=\left(x_{i}\right)_{i \in \mathbb{Z}_{+}}=x_{0} x_{1} x_{2} \ldots$,

- two-sided infinite sequences by $\bar{x}=\left(x_{i}\right)_{i \in \mathbb{Z}}=\ldots x_{-2} x_{-1} x_{0} x_{1} x_{2} \ldots$.

Let $x_{0} \ldots x_{k}$ be a finite sequence and let $\vec{y}=\left(y_{i}\right)_{i \in \mathbb{Z}_{+}}$and $\bar{z}=\left(z_{-i}\right)_{i \in \mathbb{N}}$. We set

$$
\begin{aligned}
\left(x_{0} \ldots x_{k}\right)^{\infty} & =\underbrace{x_{0} \ldots x_{k}}_{\infty \text { many times }} \underbrace{x_{0} \ldots x_{k}} \cdots, \\
x_{0} \ldots x_{k} \vec{y} & =x_{0} \ldots x_{k} y_{0} y_{1} \ldots \\
\bar{z} \vec{y} & =\ldots z_{-2} z_{-1} y_{0} y_{1} \ldots
\end{aligned}
$$


Let $X_{s}^{+}=\left\{\vec{x} \in\{0,1\}^{\mathbb{Z}_{+}}: \vec{x}\right.$ is allowed $\}$ be the space of all allowed sequences with respect to $f_{s}$. The metric $d$ on the space $X_{s}^{+}$is given as follows: For two sequences $\vec{x}=\left(x_{i}\right)_{i \in \mathbb{Z}_{+}}$and $\vec{y}=\left(y_{i}\right)_{i \in \mathbb{Z}_{+}}$, let

$$
d(\vec{x}, \vec{y})= \begin{cases}0, & \vec{x}=\vec{y}, \\ 2^{-k}, & \vec{x} \neq \vec{y},\end{cases}
$$

where $k=\min \left\{j \in \mathbb{Z}_{+}: x_{j} \neq y_{j}\right\}$ for $\vec{x} \neq \vec{y}$. Since the space of all right-infinite sequences is compact, by Proposition 2.3 one can prove that the space $X_{s}^{+}$is compact. The one-sided shift $\sigma: X_{s}^{+} \rightarrow X_{s}^{+}$given by $\sigma\left(\left(x_{i}\right)_{i \in \mathbb{Z}_{+}}\right)=\left(x_{i+1}\right)_{i \in \mathbb{Z}_{+}}$is continuous.

Since for every path in the Markov graph $I_{i_{0}} \rightarrow I_{i_{1}} \rightarrow \cdots \rightarrow I_{i_{k}} \rightarrow \cdots$ there is a point $\xi \in I_{i_{0}}$ such that $f_{s}^{k}(\xi) \in I_{i_{k}}, k \in \mathbb{N}$ ([M-S, p. 83]), for every allowed sequence $\vec{x}$ there is a point $\xi \in I^{x_{0}}$ such that $f_{s}^{k}(\xi) \in I^{x_{k}}, k \in \mathbb{N}$. Similarly to [P-Y, pp. 41-43], one can prove that there exists a continuous mapping $\pi: X_{s}^{+} \rightarrow[0,1]$ having the following properties:

(1) $\pi$ is a semi-conjugacy, i.e., $\pi$ is surjective and $\pi \circ \sigma=f_{s} \circ \pi$,

(2) points $\xi \in[0,1]$ have exactly one or two pre-images in $X_{s}^{+}$, i.e., for every $\xi \in[0,1]$, the set $E(\xi)=\left\{\vec{x} \in X_{s}^{+}: \pi(\vec{x})=\xi\right\}$ has either one or two elements,

(3) the set of points $\xi \in[0,1]$ such that $E(\xi)$ has more than one element is equal to the countable set $\bigcup_{i \in \mathbb{Z}_{+}} f_{s}^{-i}\left\{c_{s}\right\}$.

The mapping $\pi$ is given by $\pi(\vec{x})=\bigcap_{i=0}^{\infty} f_{s}^{-i}\left(I^{x_{i}}\right)$, where $\vec{x}=\left(x_{i}\right)_{i \in \mathbb{Z}_{+}} \in X_{s}^{+}$, i.e., $\pi(\vec{x})$ corresponds to the only point $\xi \in[0,1]$ such that $f_{s}^{i}(\xi) \in I^{x_{i}}$ for $i \geq 0$. It is easy to see that $E\left(c^{1}\right)$ consists of exactly one sequence. Denote this sequence by $\vec{c}_{1}=c_{1} c_{2} \ldots$ Note that $\vec{c}_{1}=\left(c_{1} \ldots c_{N}\right)^{\infty}$ in the periodic case and $\vec{c}_{1}=c_{1} \ldots c_{M-1}\left(c_{M} \ldots c_{M+N-1}\right)^{\infty}$ in the strictly preperiodic case. The two elements of $E\left(c_{s}\right)$ are $0 \vec{c}_{1}$ and $1 \vec{c}_{1}$. For every point $\xi \in[0,1]$ such that there is $k \in \mathbb{N}$ with $f_{s}^{k}(\xi)=c_{s}$ and $f_{s}^{j}(\xi) \neq c_{s}$ for $j \in \mathbb{N}, j<k$, the elements of $E(\xi)$ are $x_{0} x_{1} \ldots x_{k-1} 0 \vec{c}_{1}$ and $x_{0} x_{1} \ldots x_{k-1} 1 \vec{c}_{1}$.

Let us define an equivalence relation $\sim$ on the space $X_{s}^{+}$as follows: Two sequences $\vec{x}, \vec{y} \in X_{s}^{+}, \vec{x}=\left(x_{i}\right)_{i \in \mathbb{Z}_{+}}, \vec{y}=\left(y_{i}\right)_{i \in \mathbb{Z}_{+}}$, are equivalent, $\vec{x} \sim \vec{y}$, if either $\vec{x}=\vec{y}$, or there is $k \in \mathbb{Z}_{+}$such that

(1) $x_{i}=y_{i}, 0 \leq i<k$,

(2) $\left|x_{k}-y_{k}\right|=1$,

(3) $\sigma^{k+1}(\vec{x})=\sigma^{k+1}(\vec{y})=\vec{c}_{1}$.

The quotient map $\widetilde{\pi}: X_{s}^{+} / \sim \rightarrow[0,1]$ is defined by $\widetilde{\pi}([\vec{x}])=\pi(\vec{x})$. Note that $\vec{x}$ and $\vec{y}$ are equivalent if and only if $\pi(\vec{x})=\pi(\vec{y})$. In particular, this implies that $\widetilde{\pi}$ is a homeomorphism. We will often identify $X_{s}^{+} / \sim$ and $[0,1]$. If there is a sequence $\vec{y} \in[\vec{x}]$ with $\vec{y} \neq \vec{x}$, it is unique, and we denote it by $\vec{x}^{*}=\left(x_{i}^{*}\right)_{i \in \mathbb{Z}_{+}}$. If there is no such $\vec{y} \in[\vec{x}]$ with $\vec{y} \neq \vec{x}$, we put $\vec{x}^{*}=\vec{x}$. 
In this way we have, in fact, defined itineraries. The itinerary of a point $\xi \in[0,1]$ (with respect to $f_{s}$ ) is $[\vec{x}] \in X_{s}^{+} / \sim$ with $\pi(\vec{x})=\xi$. This definition is slightly different from the usual ones [Br-D], [C-E], but it turns out to be very useful. Note that $\left[\vec{c}_{1}\right]$ is the kneading sequence of the mapping $f_{s}$.

Lemma 2.6. If $M=0$, then $\vec{c}_{1}=\left(c_{1} \ldots c_{N}\right)^{\infty}$ and in the finite sequence $c_{1} \ldots c_{N}$ there are an even number of ones.

Proof. For the point $1 \in[0,1]$, the orbit is given by

$$
\mathcal{O}_{s}(1)=\left\{1,0, f_{s}^{2}(1), \ldots, f_{s}^{N-2}(1), c_{s}\right\} .
$$

The path in the Markov graph associated with the point 1 is $I_{N-2} \rightarrow$ $I_{i_{1}} \rightarrow I_{i_{2}} \rightarrow \cdots \rightarrow I_{i_{N-1}} \rightarrow I_{N-2}$ and the associated allowed sequence is $\left(c_{1} \ldots c_{N}\right)^{\infty}$. There is $\epsilon>0$ such that $[1-\epsilon, 1] \subset I_{N-2}, f_{s}^{N}([1-\epsilon, 1]) \subset I_{N-2}$ and $f_{s}^{j}([1-\epsilon, 1]) \subset I_{i_{j}}$ for every $1 \leq j \leq N-1$. Therefore, the first $N+1$ elements of the allowed sequence associated with the point $1-\epsilon$ are $c_{1} \ldots c_{N} c_{1}$. Since $f_{s}$ is increasing on $I^{0}$ and decreasing on $I^{1}$, and since $f_{s}^{N}$ is order-preserving on $[1-\epsilon, 1] \subset I^{1}$, we have $I_{i_{j}} \subset I^{1}$ for an odd number of $I_{i_{j}}, 1 \leq j \leq N-1$. Therefore, in the sequence $c_{1} \ldots c_{N}$ there are an even number of ones.

For a two-sided sequence $\bar{x}=\left(x_{i}\right)_{i \in \mathbb{Z}}$, we denote the right-infinite sequence $x_{j} x_{j+1} x_{j+2} \ldots$, also called a right tail of $\bar{x}$, by $\vec{x}_{j}=x_{j} x_{j+1} x_{j+2} \ldots$. A two-sided sequence $\bar{x} \in\{0,1\}^{\mathbb{Z}}$ is called allowed (with respect to $f_{s}$ ) if all of its right tails $\vec{x}_{j}$ are allowed. An immediate consequence of Proposition 2.3 is that a two-sided sequence $\bar{x}=\left(x_{i}\right)_{i \in \mathbb{Z}}$ is allowed if and only if for every $k \in \mathbb{N}, k \geq(M+N-1) / 2$, the finite sequence $x_{-k} \ldots x_{k}$ is allowed. Moreover, when $M=0$, from Proposition 2.5 it follows that a two-sided sequence $\bar{x}=\left(x_{i}\right)_{i \in \mathbb{Z}}$ is allowed if and only if all of its finite parts of length $N+1$ are allowed, i.e. if and only if, for every $j \in \mathbb{Z}$, the finite sequence $x_{j} x_{j+1} \ldots x_{j+N}$ is allowed.

Let $X_{s}=\left\{\bar{x} \in\{0,1\}^{\mathbb{Z}}: \bar{x}\right.$ is allowed $\}$ denote the space of all allowed two-sided sequences with respect to $f_{s}$. The metric $d$ on the space $X_{s}$ is given as follows: For two sequences $\bar{x}, \bar{y} \in X_{s}, \bar{x}=\left(x_{i}\right)_{i \in \mathbb{Z}}, \bar{y}=\left(y_{i}\right)_{i \in \mathbb{Z}}$, if $\bar{x} \neq \bar{y}$, let $k=\min \left\{|j|: j \in \mathbb{Z}, x_{j} \neq y_{j}\right\}$. Then

$$
d(\bar{x}, \bar{y})= \begin{cases}0, & \bar{x}=\bar{y} \\ 2^{-k}, & \bar{x} \neq \bar{y} .\end{cases}
$$

The shift map $\sigma: X_{s} \rightarrow X_{s}$ given by $(\sigma \bar{x})_{i}=x_{i+1}$ for every $i \in \mathbb{Z}$ is a homeomorphism ([P-Y, p. 2]). Let us define an equivalence relation $\approx$ on the space $X_{s}$ as follows: Two sequences $\bar{x}, \bar{y} \in X_{s}, \bar{x}=\left(x_{i}\right)_{i \in \mathbb{Z}}, \bar{y}=$ $\left(y_{i}\right)_{i \in \mathbb{Z}}$, are equivalent, $\bar{x} \approx \bar{y}$, if there is $k \in \mathbb{Z}$ with $x_{i}=y_{i}$ for $i<k$, and $x_{k} x_{k+1} x_{k+2} \ldots \sim y_{k} y_{k+1} y_{k+2} \ldots$ This enables us to obtain, similarly to the proof of Proposition 2 in [B], the following assertion, also resembling 
Theorem 2.5 in [Br-D]: There is a homeomorphism $h: X_{s} / \approx \rightarrow C_{s}$ such that $h(\widetilde{\sigma}([\bar{x}]))=\widehat{f}_{s}(h([\bar{x}]))$ for every $[\bar{x}] \in X_{s} / \approx$, where $\widetilde{\sigma}: X_{s} / \approx \rightarrow X_{s} / \approx$ is given by $\widetilde{\sigma}([\bar{x}])=[\sigma \bar{x}]$, and $\widehat{f}_{s}: C_{s} \rightarrow C_{s}$ is given by $\widehat{f}_{s}\left(\ldots, \xi_{-3}, \xi_{-2}, \xi_{-1}\right)=$ $\left(\ldots, \xi_{-2}, \xi_{-1}, f_{s}\left(\xi_{-1}\right)\right)$, i.e., the maps $\widetilde{\sigma}$ and $\widehat{f}_{s}$ are conjugate. Note that the maps $\widetilde{\sigma}$ and $\hat{f}_{s}$ are homeomorphisms. We will often identify $C_{s}$ and $X_{s} / \approx$.

If there is a sequence $\bar{y} \in[\bar{x}]$ with $\bar{y} \neq \bar{x}$, it is unique, and we denote it by $\bar{x}^{*}=\left(x_{i}^{*}\right)_{i \in \mathbb{Z}}$. If there is no such $\bar{y} \in[\bar{x}]$ with $\bar{y} \neq \bar{x}$, we put $\bar{x}^{*}=\bar{x}$. Let $\pi_{j}: X_{s} / \approx \rightarrow[0,1], j \in \mathbb{Z}_{+}$, be the projection on the $j$ th coordinate, i.e. $\pi_{j}[\bar{x}]=\pi\left(\vec{x}_{-j}\right)$.

For a two-sided sequence $\bar{x}=\left(x_{i}\right)_{i \in \mathbb{Z}}$, we denote the left-infinite sequence $\ldots x_{j-1} x_{j}$, also called a left tail of $\bar{x}$, by $\overleftarrow{x}_{j}=\ldots x_{j-1} x_{j}$. A left-infinite sequence $\overleftarrow{x}=\left(x_{-i}\right)_{i \in \mathbb{N}}$ is allowed if for every $k \in \mathbb{N}, k \geq M+N$, the finite sequence $x_{-k} \ldots x_{-1}$ is allowed. Moreover, when $M=0$, a left-infinite sequence is allowed if all of its finite parts of length $N+1$ are allowed. Note that if $\bar{x}$ is allowed, then all of its left tails $\overleftarrow{x}_{j}$ are allowed.

Similarly to Proposition 3 in [B] and Corollary 2.10 in [Br-D], the following assertion is obtained: Each left-infinite sequence $\overleftarrow{x}=\ldots x_{-2} x_{-1}$ describes one composant in $C_{s}$, which is the set of two-sided sequences having a left tail common to $\overleftarrow{x}$. Two sequences $\overleftarrow{x}$ and $\overleftarrow{y}$ describe the same composant if and only if they have a common left tail.

Every composant of $C_{s}$ is arcwise connected. Let $\overleftarrow{a}=\ldots a_{-2} a_{-1}$ and $n \in \mathbb{Z}_{+}$. The set

$$
A_{\bar{a}}^{n}=\left\{[\bar{x}] \in C_{s}: \exists \bar{x} \in[\bar{x}], \overleftarrow{x}_{-n}=\overleftarrow{a}\right\}
$$

is an arc and we call it a basic arc. For a fixed left-infinite sequence $\overleftarrow{y}=$ $\ldots y_{-2} y_{-1}$, let $C$ be the corresponding composant of $C_{s}$. If $A_{\bar{v}}^{n}$ is a basic arc contained in the composant $C$, then either $\overleftarrow{v}_{-1}=\overleftarrow{y}_{-n}$, or there is $k \in \mathbb{N}$ with $v_{-k} \neq y_{-n-k+1}$ and $\overleftarrow{v}_{-k-1}=\overleftarrow{y}_{-n-k}$. In the first case we put $k=0$. Whenever it is clear which sequence $\overleftarrow{y}$ represents the composant containing the basic $\operatorname{arc} A_{\bar{v}}^{n}$, and when $k=0$, we write, for simplicity, only $A^{n}$ instead of $A_{\bar{y}_{-n}}^{n}$. When $k>0$, we write, for simplicity, only $A_{v}^{n}$ instead of $A_{\bar{v}}^{n}$, where $v=v_{-k} \ldots v_{-1}$, and we understand that $\overleftarrow{v}_{-k-1}=\overleftarrow{y}_{-n-k}$

We now introduce an order structure on the composant $C$.

Definition 2.7. For $n \in \mathbb{N}$, let $P(n)=\operatorname{card}\left\{i: y_{-i}=1,1 \leq i \leq n\right\}$. If $n=0$, let $P(0)=0$. An arc $A^{n}$ is called even (respectively odd) if $P(n)$ is even (respectively odd). An $\operatorname{arc} A_{v}^{n}, v=v_{-k} \ldots v_{-1}, v_{-k} \neq y_{-n-k}$, is called even (respectively odd) if $(-1)^{P(n+k)}=\prod_{i=1}^{k}(-1)^{v_{-i}}$ (respectively $\left.(-1)^{P(n+k)} \neq \prod_{i=1}^{k}(-1)^{v_{-i}}\right)$.

Definition 2.8. The generalized parity-lexicographical ordering $\preceq$ on $C$ is defined as follows: For $[\bar{x}],[\bar{z}] \in C$, let $k=k([\bar{x}],[\bar{z}])=\max \{i \in \mathbb{N}$ : 
$x_{-i} \neq y_{-i}$ or $\left.z_{-i} \neq y_{-i}, \bar{x}=\left(x_{i}\right)_{i \in \mathbb{Z}} \in[\bar{x}], \bar{z}=\left(z_{i}\right)_{i \in \mathbb{Z}} \in[\bar{z}]\right\}$. If $x_{-i}=y_{-i}$ and $z_{-i}=y_{-i}$ for all $i \in \mathbb{N}, \bar{x} \in[\bar{x}], \bar{z} \in[\bar{z}]$, set $k=0$. We say that $\bar{x} \prec \bar{z}$ if either $(-1)^{P(k)} x_{-k}<(-1)^{P(k)} z_{-k}$, or there exists $l \in \mathbb{Z}, l>-k$, such that $x_{i}=z_{i}$ for $-k \leq i<l$ and

$$
(-1)^{P(k)} \varepsilon x_{l}<(-1)^{P(k)} \varepsilon z_{l},
$$

where $\varepsilon=\prod_{i=-k}^{l-1}(-1)^{x_{i}}=\prod_{i=-k}^{l-1}(-1)^{z_{i}} \in\{-1,1\}$. We say that $[\bar{x}] \preceq[\bar{z}]$ if $\bar{x} \prec \bar{z}$ or $\bar{x}=\bar{z}$.

Note that the ordering depends on the left-infinite sequence $\overleftarrow{y}$ chosen. The choice of another representative of this particular composant would lead either to the same, or to the opposite ordering. If $C$ is a composant without endpoints, then there exists an order-preserving bijection $\phi$ between the real line, endowed with its natural order, and $C$, endowed with the ordering $\preceq$. If $C$ has one endpoint, then there exists an order-preserving bijection $\psi$ between the half-line, endowed with its natural order, and $C$, endowed with the ordering $\preceq$. Therefore, the ordering $\preceq$ on the composant $C$ is natural. Note that $\phi$ and $\psi$ are continuous, but their inverses are not.

In order to describe the structure of composants, let us define some special points.

Definition 2.9. A point $[\bar{x}] \in C_{s}$ is called an identification point or briefly an $i$-point if there is $m \in \mathbb{Z}_{+}$with $\vec{x}_{-m+1}=\vec{c}_{1}$. Let $[\bar{x}] \in C_{s}$ be an $i$ point with $\bar{x} \neq \bar{x}^{*}$. The level of $[\bar{x}]$ is defined by $L[\bar{x}]=m$ if $\left|x_{-m}-x_{-m}^{*}\right|=1$. If $\bar{x}=\bar{x}^{*}$, let $L[\bar{x}]=\infty$.

The meaning of the $i$-points and their levels is visible from the following: Let $\overleftarrow{a}=\left(a_{-i}\right)_{i \in \mathbb{N}}$ and $\overleftarrow{b}=\left(b_{-i}\right)_{i \in \mathbb{N}}, \overleftarrow{a} \neq \overleftarrow{b}$, be allowed sequences. For $n \in \mathbb{N}$, let $A_{\bar{a}}^{n}$ and $A_{\bar{b}}^{n}$ be the basic arcs. If there is $[\bar{x}] \in A_{\bar{a}}^{n} \cap A_{\bar{b}}^{n}$, then $\overleftarrow{x}_{-n}=\overleftarrow{a}$ and $\overleftarrow{x}_{-n}^{*}=\overleftarrow{b}$. Hence, $[\bar{x}]$ is an $i$-point, and there is $m \geq n$ with $x_{-i}=x_{-i}^{*}=a_{n-i-1}$ for $i>m,\left|x_{-m}-x_{-m}^{*}\right|=1$ and $\vec{x}_{-m+1}=\vec{x}_{-m+1}^{*}=\vec{c}_{1}$, implying that $L[\bar{x}]=m$. Also, if $[\bar{y}] \in A_{\bar{a}}^{n}$ is an $i$-point with $L[\bar{y}]>n$, then $[\bar{y}] \in \partial A_{\bar{a}}^{n}$. Therefore, the basic arcs $A_{\bar{a}}^{n}$ and $A_{\bar{b}}^{n}$ are neighboring if and only if there is $k \geq n$ with:

- $a_{-i}=b_{-i}$ for $i>k$,

- $\left|a_{-k}-b_{-k}\right|=1$,

- $a_{-k+i}=b_{-k+i}=c_{i}$ for $1 \leq i \leq k-1$.

Note that if $A_{\bar{a}}^{n}$ has boundary points $[\bar{x}]$ and $[\bar{y}]$ with $L[\bar{x}]=l$ and $L[\bar{y}]=k$, then $\left.\pi_{n-1}\right|_{A_{\bar{a}}^{n}}$ is an injection and $\pi_{n-1}\left(A_{\bar{a}}^{n}\right)=\left\{\pi_{n-1}[\bar{x}]:[\bar{x}] \in A_{\bar{a}}^{n}\right\}$ is a closed interval with boundary points $c^{l-n+1}$ and $c^{k-n+1}$. Let $A_{\bar{b}}^{n}$ be another basic arc. Let $\left\{\left[\bar{x}^{0}\right] \prec \cdots \prec\left[\bar{x}^{i}\right]\right\}$ be the ordered set of all $i$-points of $A_{\bar{a}}^{n}$, and $\left\{\left[\bar{u}^{0}\right] \prec \cdots \prec\left[\bar{u}^{j}\right]\right\}$ be the ordered set of all $i$-points of $A_{\bar{b}}^{n}$. If 
$\pi_{n-1}\left(\partial A_{\bar{a}}^{n}\right)=\pi_{n-1}\left(\partial A_{\bar{b}}^{n}\right)$, then $i=j$ and either $L\left[\bar{x}^{m}\right]=L\left[\bar{u}^{m}\right]$ for every $m \in\{1, \ldots, j-1\}$ if $A_{\bar{a}}^{n}$ and $A_{\bar{b}}^{n}$ have the same parity, or $L\left[\bar{x}^{m}\right]=L\left[\bar{u}^{j-m}\right]$ for every $m \in\{1, \ldots, j-1\}$ if they have different parity. For every $k \in$ $\{0, \ldots, n-1\}$, the arc $A_{\overleftarrow{a}}^{n}$ is a union of $\operatorname{arcs} A_{w}^{k}$, i.e. $A_{\overleftarrow{a}}^{n}=\bigcup_{w} A_{w}^{k}$, where $w$ is a finite sequence of length $n-k$ such that $\overleftarrow{a} w$ is allowed. Since $f_{s}$ is l.e.o. and $\pi \circ \sigma=f_{s} \circ \pi$, for every arc $A$ there is $m \in \mathbb{Z}_{+}$such that $\tilde{\sigma}^{m}(A)=\left\{\widetilde{\sigma}^{m}[\bar{x}]:[\bar{x}] \in A\right\}$ contains at least one $i$-point.

Let us prove some additional important properties of basic arcs.

Proposition 2.10. Let $\overleftarrow{a}=\left(a_{-i}\right)_{i \in \mathbb{N}}$ be an allowed sequence, $n \in \mathbb{N}$, and let $A_{\bar{a}}^{n}$ be the associated basic arc. Then, for every $i$-point $[\bar{y}] \in \operatorname{int} A_{\bar{a}}^{n}$, there are points $[\bar{x}],[\bar{z}] \in A_{\bar{a}}^{n},[\bar{x}] \prec[\bar{y}] \prec[\bar{z}]$, such that, for every point $[\bar{u}] \in A_{\bar{a}}^{n},[\bar{x}] \preceq[\bar{u}] \prec[\bar{y}]$, there is a point $[\bar{v}] \in A_{\bar{a}}^{n},[\bar{y}] \prec[\bar{v}] \preceq[\bar{z}]$, such that $\left[\vec{u}_{-l+1}\right]=\left[\vec{v}_{-l+1}\right]$, where $l=L[\bar{y}]$.

We say that the arc $A_{\bar{a}}^{n}$ is $[\bar{y}]$-symmetric between $[\bar{x}]$ and $[\bar{z}]$. If either $[\bar{x}] \in \partial A_{\bar{a}}^{n}$ or $[\bar{z}] \in \partial A_{\bar{a}}^{n}$, we say that the $\operatorname{arc} A_{\bar{a}}^{n}$ is $[\bar{y}]$-symmetric.

Proof. Let $A_{\bar{a}}^{n}$ be a basic arc, $[\bar{y}] \in \operatorname{int} A_{\bar{a}}^{n}$ and $L[\bar{y}]=l$. Then $l<n$ and $[\bar{y}] \in \operatorname{int} A_{\bar{y}_{-l-1}}^{l+1} \subseteq \operatorname{int} A_{\bar{a}}^{n}$. Let $J=\pi_{l}\left(A_{\bar{y}_{-l-1}}^{l+1}\right) \subseteq[0,1]$. Then $c_{s} \in \operatorname{int} J$. The map $f_{s}$ is symmetric on the closed interval $[0, b]$, where $0 \neq b \in f_{s}^{-1}\left(f_{s}(0)\right)$, with the point $c_{s}$ as the center of symmetry. Therefore, there is a closed interval $L \subseteq J$ such that $f_{s}$ is symmetric on $L$. Let $A=\pi_{l}^{-1}(L) \cap A_{\bar{y}_{-l-1}}^{l+1}$. Then $A=\left\{[\bar{x}] \in A_{\bar{y}_{-l-1}}^{l+1}: \widetilde{\pi}\left[\vec{x}_{-l}\right] \in L\right\}$ and for every point $[\bar{u}] \in A,\left[\vec{u}_{-l}\right] \prec$ $\left[0 \vec{c}_{1}\right]$, there is a point $[\bar{v}] \in A,\left[0 \vec{c}_{1}\right] \prec\left[\vec{v}_{-l}\right]$, such that $\left[\vec{u}_{-l+1}\right]=\left[\vec{v}_{-l+1}\right]$. Therefore, $[\bar{x}],[\bar{z}] \in \partial A$. If $[\bar{y}]$ is such that $l=\max \left\{k \in \mathbb{Z}_{+}: L[\bar{x}]=k\right.$, $\left.[\bar{x}] \in \operatorname{int} A_{\bar{a}}^{n}\right\}$, then either $[\bar{x}] \in \partial A_{\bar{a}}^{n}$ or $[\bar{z}] \in \partial A_{\bar{a}}^{n}$.

Note that, if the basic arc $A_{\bar{a}}^{n}$ contains an $i$-point $[\bar{y}]$ such that $L[\bar{y}]=$ $n-1$, then $A_{\bar{a}}^{n}$ is $[\bar{y}]$-symmetric. If $A_{\bar{a}}^{n}$ is $[\bar{y}]$-symmetric and $[\bar{x}] \in \partial A_{\bar{a}}^{n}$ then in a periodic case $[\bar{z}]$ is an $i$-point, and in a strictly preperiodic case $[\bar{z}]$ is not an $i$-point.

Every basic arc contains finitely many $i$-points for which the following direct consequence of the previous proposition is valid:

Corollary 2.11. Let $\left\{\left[\bar{x}^{0}\right] \prec\left[\bar{x}^{1}\right] \prec \cdots \prec\left[\bar{x}^{m}\right]\right\}$ be the set of all $i$-points of the basic arc $A_{\bar{a}}^{n}$. Let $k \in\{1, \ldots, m-1\}$ and $j \in \mathbb{N}, j \leq \min \{k, m-k\}$, be such that $A_{\bar{a}}^{n}$ is $\left[\bar{x}^{k}\right]$-symmetric between $\left[\bar{x}^{k-j}\right]$ and $\left[\bar{x}^{k+j}\right]$. Then $L\left[\bar{x}^{k-i}\right]=$ $L\left[\bar{x}^{k+i}\right]$ for every $i \in\{1, \ldots, j-1\}$.

COROllary 2.12. Let $A_{\bar{a}}^{n}$ and $A_{\bar{b}}^{n}$ be two neighboring arcs, let $\left\{\left[\bar{x}^{0}\right] \prec\right.$ $\left.\left[\bar{x}^{1}\right] \prec \cdots \prec\left[\bar{x}^{m}\right]\right\}$ be their $i$-points and let $k \in\{1, \ldots, m-1\}$ be such that $\left[\bar{x}^{k}\right]=A_{\bar{a}}^{n} \cap A_{\bar{b}}^{n}$. Let $j=\min \{k, m-k\}$. Then for every $[\bar{u}],\left[\bar{x}^{k-j}\right] \preceq$ 
$[\bar{u}] \prec\left[\bar{x}^{k}\right]$, there is $[\bar{v}],\left[\bar{x}^{k}\right] \prec[\bar{v}] \preceq\left[\bar{x}^{k+j}\right]$, such that $\left[\vec{u}_{-n+1}\right]=\left[\vec{v}_{-n+1}\right]$. In particular, $L\left[\bar{x}^{k-i}\right]=L\left[\bar{x}^{k+i}\right]$ for every $i \in \mathbb{N}, i \leq j-1$.

We say that the neighboring arcs $A_{\bar{a}}^{n}$ and $A_{\bar{b}}^{n}$ are $\left[\bar{x}^{k}\right]$-symmetric. If $k=m-k$, we say that the $\operatorname{arcs} A_{\bar{a}}^{n}$ and $A_{\bar{b}}^{n}$ are $n$-symmetric.

Proof. Since $\left[\bar{x}^{k}\right]=A_{\bar{a}}^{n} \cap A_{\bar{b}}^{n}$, we have $l=L\left[\bar{x}^{k}\right] \geq n, A_{\bar{a}}^{n} \cap A_{\bar{b}}^{n} \subseteq A_{\overleftarrow{x}_{-l-1}^{k}}^{l+1}$ and the statement is a direct consequence of Proposition 2.10.

EXAmple 2.13. Let $M=0$ and $N=3$. There is only one mapping $f_{s}$ with periodic extreme points of period three. For this mapping $X_{s}^{+}=$ $\left\{\left(x_{i}\right)_{i \in \mathbb{Z}_{+}}: x_{j} x_{j+1} \neq 00, \forall j \in \mathbb{Z}_{+}\right\}$, its kneading sequence is $\vec{c}_{1}=(101)^{\infty}$ and the equivalence relation is given by $0(101)^{\infty} \sim 1(101)^{\infty}=(110)^{\infty}$. The corresponding continuum is $C_{s}$. For the two-sided sequence $\bar{x}=$ $\ldots x_{-2} x_{-1} \cdot x_{0} x_{1} \ldots=\ldots 10110111.0(110)^{\infty}$, the point $[\bar{x}]$ of the continuum $C_{s}$ is an $i$-point, $\bar{x}^{*}=\ldots 10110101.0(110)^{\infty}$ and $L[\bar{x}]=2$. From now on, we will write the point $[\bar{x}]$ as $\ldots 101101 \frac{1}{0} 1.0(110)^{\infty}$, since this notation is simple and it provides all the information concerning the $i$-point $[\bar{x}]$, both representatives and the level.

Let the sequence $\overleftarrow{y}=\ldots 10110110$ represent the composant $C$ of $C_{s}$. Let $\overleftarrow{a}=\ldots 101111$ and $n=3$. Then the $\operatorname{arc} A_{\bar{a}}^{3}=\left\{[\bar{x}] \in C_{s}: \exists \bar{x} \in[\bar{x}]\right.$, $\left.\overleftarrow{x}_{-3}=\overleftarrow{a}\right\}$ is contained in $C$ and it is even. All allowed finite sequences $w$ of length 3 are $011 \prec 010 \prec 110 \prec 111 \prec 101$, and for every $w$, the sequence $\overleftarrow{a} w$ is allowed. Therefore, the $i$-points contained in $A_{\overleftarrow{a}}^{3}$ are as follows:

$$
\begin{aligned}
{[\bar{u}] } & =\ldots 1011 \frac{0}{1} 101 \cdot(101)^{\infty} \prec \ldots 10111101 \cdot \frac{1}{0}(101)^{\infty} \\
& \prec \ldots 101111 \frac{0}{1} 1 \cdot(011)^{\infty} \prec \ldots 10111111 \cdot \frac{0}{1}(101)^{\infty} \\
& \prec \ldots 1011111 \frac{1}{0} \cdot(101)^{\infty} \prec \ldots 10111 \frac{1}{0} 10 \cdot(110)^{\infty}=[\bar{v}],
\end{aligned}
$$

and $\partial\left(A_{\bar{a}}^{3}\right)=\{[\bar{u}],[\bar{v}]\}$. Since $L[\bar{u}]=4$ and $L[\bar{v}]=3$, we have $\pi_{2}\left(A_{\bar{a}}^{3}\right)=$ $\left[c^{4-3+1}, c^{3-3+1}\right]=\left[c^{2}, c^{1}\right]=[0,1]$. Neighboring arcs of the arc $A_{\bar{a}}^{3}$ are $A_{\bar{u}}^{3}$ and $A_{\overleftarrow{v}}^{3}$ with $\overleftarrow{u}=\ldots 101101$ and $\overleftarrow{v}=\ldots 101110$

3. Structure of the composants. In order to distinguish the composants of the continuum $C_{s}$ with folding points from those without folding points, let us first determine the folding points of $C_{s}$.

The $\omega$-limit set of $\xi \in[0,1]$ is the set of accumulation points of the orbit of $\xi$, i.e., $\omega(\xi)=\left\{\zeta \in[0,1]: \exists\right.$ a sequence $n_{i} \rightarrow \infty$ with $\left.f_{s}^{n_{i}}(\xi)=\zeta\right\}$ ([M-S, p. 555]). Note that because $\mathcal{O}_{s}\left(c_{s}\right)$ is finite, $\omega\left(c_{s}\right)$ is the periodic orbit which $c_{s}$ belongs to or is eventually mapped to. Therefore, the folding points of $C_{s}$ are $\bar{c}^{j}=\left[\bar{c}^{j}\right], j \in \mathbb{N}, j \geq M$, where $\bar{c}^{j}=\left(c_{i}\right)_{i \in \mathbb{Z}}$ is such that $c_{k N} \ldots c_{k N+N-1}=c_{j} \ldots c_{j+N-1}$ for every $k \in \mathbb{Z}$ (cf. [Brn2]). Note that $\bar{c}^{j+i N}=\bar{c}^{j}$ for every $i \in \mathbb{Z}_{+}$. Therefore, $C_{s}$ has $N$ folding points and $N$ different composants containing one folding point each. If $c_{s}$ is periodic, 
these points are actually the endpoints of $C_{s}$. Note that endpoints of $C_{s}$ are $i$-points, $L\left(\bar{c}^{j}\right)=\infty$ for every $j \in\{0, \ldots, N-1\}$, and these are the only $i$-points in $C_{s}$ with this property.

Firstly, we are interested in the structure of composants of folding points. Since $\widetilde{\sigma}: C_{s} \rightarrow C_{s}$ is a homeomorphism which permutes composants of folding points, it is sufficient to describe the structure of the composant of the folding point $\bar{c}^{K}$ such that $K \geq M$ and $K=k 2 N$ for some $k \in \mathbb{Z}_{+}$. From now on, we denote it by $C$. In [K1] and [K2], L. Kailhofer described some properties of the composant of the endpoint $\bar{c}=\left[\bar{c}^{0}\right]=\left[\bar{c}^{N}\right]$, and in this section there are some objects and results similar to those in [K1] and [K2]. From now on, let a representative of the composant $C$ be the sequence $\overleftarrow{c}^{K}=\left(c_{-i}\right)_{i \in \mathbb{N}}$ with $c_{-i N-N-1} \ldots c_{-i N-1}=c_{-N+K-1} \ldots c_{K-1}$ for every $i \in \mathbb{Z}_{+}$. Then the ordering $\preceq$ on $C$ is unique. It is easy to see that, for every $j \in \mathbb{N}$, the map $\widetilde{\sigma}^{j K}: C \rightarrow C$ is an order-preserving homeomorphism. In the periodic case, by Lemma 2.6 , for every $j \in \mathbb{N}$, the map $\widetilde{\sigma}^{j N}: C \rightarrow C$ is an order-preserving homeomorphism. Also, for the periodic case, $\vec{c}_{0}=\vec{c}_{N}$ and, for simplicity, we will often write only $\vec{c}$ instead of $\vec{c}_{0}$. From now on, let $K=N$ in the periodic case.

In order to describe the structure of the composant $C$, let us sort the $i$-points of $C$ in the following way: For every $p \in \mathbb{Z}_{+}$the $i$-point $[\bar{x}] \in C$ is called a p-point if there is $m \in \mathbb{Z}_{+}$with $\left[\vec{x}_{-p K-m+1}\right]=\left[\vec{c}_{1}\right]$. A $p$-point $[\bar{x}]$ has $p$-level $L_{p}[\bar{x}]=m$ if $\left|x_{-p K-m}-x_{-p K-m}^{*}\right|=1$. For every $p, m \in \mathbb{Z}_{+}$,

$$
E_{p, m}=\left\{[\bar{x}] \in C:\left|x_{-p K-m}-x_{-p K-m}^{*}\right|=1\right\}
$$

is the set of all $p$-points of level $m$, and $E_{p}=\bigcup_{m=0}^{\infty} E_{p, m} \cup\left\{\bar{c}^{K}\right\}$ is the set of all $p$-points of $C$. Set $L_{p}\left(\bar{c}^{K}\right)=\infty$ for every $p \in \mathbb{Z}_{+}$. Note that $E_{p+1} \subset E_{p}$ and $\bar{c}^{K} \in E_{p}$ for every $p \in \mathbb{Z}_{+}$. Since in the strictly preperiodic case, there is an order-preserving bijection from $(\mathbb{Z}, \leq)$ to $\left(E_{p}, \preceq\right)$ such that $0 \in \mathbb{Z}$ is mapped to $\bar{c}^{K} \in E_{p}$, from now on, the points of $E_{p}$ will be indexed by $\mathbb{Z}$.

Let $p \in \mathbb{Z}_{+}$. Let $C^{+}=\left\{[\bar{x}] \in C:\left[\bar{x}^{0}\right] \preceq[\bar{x}]\right\}$ and $E_{p}^{+}=\left\{\left[\bar{x}^{0}\right],\left[\bar{x}^{1}\right], \ldots\right\}$. Note that $\left[\bar{x}^{0}\right]=\bar{c}^{K}$. In the periodic case $C=C^{+}$and there is an orderpreserving bijection from $\left(\mathbb{Z}_{+}, \leq\right)$to $\left(E_{p}, \preceq\right)$. Therefore, the points of $E_{p}$ will be indexed by $\mathbb{Z}_{+}$and we can put $E_{p}=E_{p}^{+}$. The sequence $L_{p}\left[\bar{x}^{0}\right], L_{p}\left[\bar{x}^{1}\right], \ldots$ is called the folding pattern of the composant $C$. Let $q \in \mathbb{Z}_{+}, q>p$, and $E_{q}^{+}=\left\{\left[\bar{y}^{0}\right],\left[\bar{y}^{1}\right], \ldots\right\}$. Since $\widetilde{\sigma}^{(q-p) K}$ is an order-preserving homeomorphism of $C$, it is easy to see that, for every $i \in \mathbb{Z}_{+}$, one has $\widetilde{\sigma}^{(q-p) K}\left(\left[\bar{x}^{i}\right]\right)=\left[\bar{y}^{i}\right]$ and $L_{p}\left[\bar{x}^{i}\right]=L_{q}\left[\bar{y}^{i}\right]$. Therefore, the folding pattern of the composant $C$ does not depend on $p$. A similar sequence is also defined in [Brn3].

Example 3.1. Periodic case. Let $f_{s}$ be the mapping with the periodic kneading sequence $\vec{c}_{1}=(101)^{\infty}$ as in Example 2.13 and let $C_{s}$ be the corresponding continuum. Then $C_{s}$ has three endpoints: $\bar{c}=\ldots 110110 .(110)^{\infty}$, 


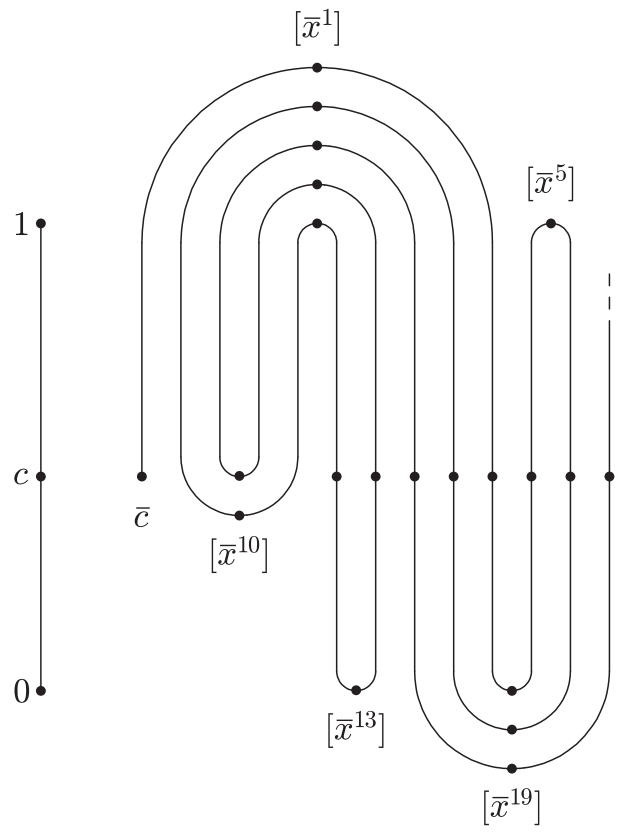

\begin{tabular}{|c|c|c|}
\hline$E_{0}$ & $L_{0}$ & $L_{1}$ \\
\hline $\bar{c}=\ldots 110110 .(110)^{\infty}$ & $\infty$ & $\infty$ \\
\hline$\left[\bar{x}^{1}\right]=\ldots 11011 \frac{0}{1} \cdot(101)^{\infty}$ & 1 & \\
\hline$\left[\bar{x}^{2}\right]=\ldots 110111 \cdot \frac{1}{0}(101)^{\infty}$ & 0 & \\
\hline$\left[\bar{x}^{3}\right]=\ldots 1101 \frac{1}{0} 1 .(011)^{\infty}$ & 2 & \\
\hline$\left[\bar{x}^{4}\right]=\ldots 110101 \cdot \frac{0}{1}(101)^{\infty}$ & 0 & \\
\hline$\left[\bar{x}^{5}\right]=\ldots 11 \frac{0}{1} 101 .(101)^{\infty}$ & 4 & 1 \\
\hline$\left[\bar{x}^{6}\right]=\ldots 111101 \cdot \frac{1}{0}(101)^{\infty}$ & 0 & \\
\hline$\left[\bar{x}^{7}\right]=\ldots 1111 \frac{0}{1} 1 .(011)^{\infty}$ & 2 & \\
\hline$\left[\bar{x}^{8}\right]=\ldots 111111 \cdot \frac{0}{1}(101)^{\infty}$ & 0 & \\
\hline$\left[\bar{x}^{9}\right]=\ldots 11111 \frac{1}{0} \cdot(101)^{\infty}$ & 1 & \\
\hline$\left[\bar{x}^{10}\right]=\ldots 111 \frac{1}{0} 10 \cdot(110)^{\infty}$ & 3 & 0 \\
\hline$\left[\bar{x}^{11}\right]=\ldots 11101 \frac{0}{1} \cdot(101)^{\infty}$ & 1 & \\
\hline$\left[\bar{x}^{12}\right]=\ldots 111011 \cdot \frac{1}{0}(101)^{\infty}$ & 0 & \\
\hline$\left[\bar{x}^{13}\right]=\ldots 1 \frac{1}{0} 1011 .(011)^{\infty}$ & 5 & 2 \\
\hline$\left[\bar{x}^{14}\right]=\ldots 101011 \cdot \frac{0}{1}(101)^{\infty}$ & 0 & \\
\hline$\left[\bar{x}^{15}\right]=\ldots 10101 \frac{1}{0} \cdot(101)^{\infty}$ & 1 & \\
\hline$\left[\bar{x}^{16}\right]=\ldots 101 \frac{0}{1} 10 \cdot(110)^{\infty}$ & 3 & 0 \\
\hline
\end{tabular}

Fig. 1. Composant $C$ and its $p$-points

$\bar{c}^{1}=\ldots$ 101101.(101) $)^{\infty}$ and $\bar{c}^{2}=\ldots$ 011011.(011) $)^{\infty}$. Denote the composant of the endpoint $\bar{c}$ by $C$. The 0-points of $C$, i.e., the points of the set $E_{0}$, are shown in Figure 1. Since $E_{p} \subset E_{0}$ for every $p \in \mathbb{N}$, we can say that "all" $p$-points are shown in the figure.

Strictly preperiodic case. Let now $f_{s}$ be the mapping with the strictly preperiodic kneading sequence $\vec{c}_{1}=10(01)^{\infty}$ as in Example 2.2 and let $C_{s}$ be the corresponding continuum. Then $C_{s}$ has two folding points: $\bar{c}^{3}=(01)^{\infty}$ and $\bar{c}^{4}=(10)^{\infty}$. Denote the composant of the folding point $\bar{c}^{4}$ by $C$. The 0 -points of $C$, i.e., the points of the set $E_{0}$, are shown in Figure 2. Since $E_{p} \subset E_{0}$ for every $p \in \mathbb{N}$, we can say that, as in the periodic case, "all" $p$-points are shown.

Now, we give some basic properties of the folding pattern of the composant $C$. Let $\left[\bar{x}^{n}\right] \in E_{p}^{+}$and $L_{p}\left[\bar{x}^{n}\right]=i K+k$ for some $i \in \mathbb{N}$ and $k \in \mathbb{Z}_{+}$, $k<K$. Then, for every $j \in \mathbb{Z}_{+}, j<i$, there is $\left[\bar{x}^{m}\right] \in E_{p}^{+},\left[\bar{x}^{m}\right] \prec\left[\bar{x}^{n}\right]$, such that $L_{p}\left[\bar{x}^{m}\right]=j K+k$. This holds because, for every $j \in \mathbb{Z}_{+}, j<i$, and $E_{p+i-j}^{+}=\left\{\left[\bar{z}^{0}\right],\left[\bar{z}^{1}\right], \ldots\right\} \subset E_{p}^{+}$, there is $m \in \mathbb{N}, m<n$, with $\left[\bar{x}^{n}\right]=\left[\bar{z}^{m}\right]$ and $L_{p}\left[\bar{x}^{m}\right]=L_{p+i-j}\left[\bar{z}^{m}\right]=L_{p+i-j}\left[\bar{x}^{n}\right]=j K+k$. Also, let $p, q, k \in \mathbb{Z}_{+}$ and let $\operatorname{arcs} A, B \subset C^{+}$be such that there are no $i$-points $[\bar{x}] \in \operatorname{int} A$ and $[\bar{y}] \in \operatorname{int} B$ with $L_{p}[\bar{x}]>k$ and $L_{q}[\bar{y}]>k$. Let $\pi_{p K+k}(A)=\pi_{q K+k}(B)$ and let 


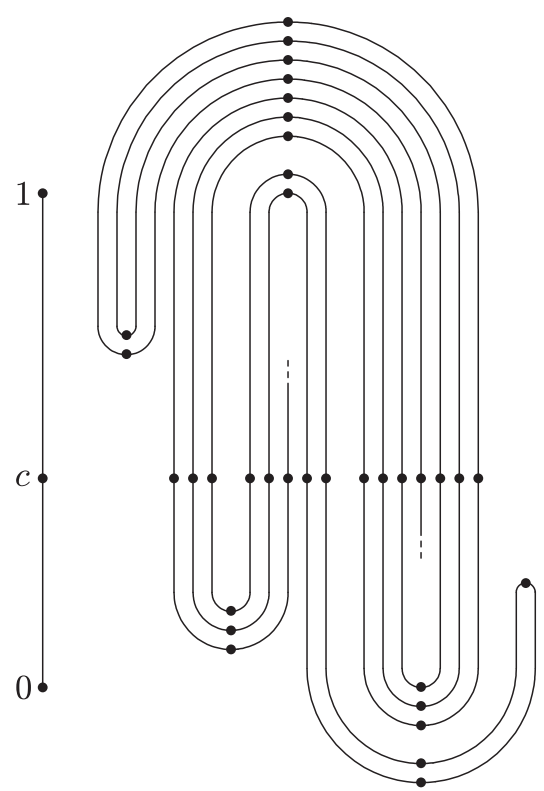

\begin{tabular}{|c|c|c|}
\hline$E_{0}$ & $L_{0}$ & $L_{1}$ \\
\hline $\bar{c}^{4}=\ldots 10101010 .(10)^{\infty}$ & $\infty$ & $\infty$ \\
\hline$\left[\bar{x}^{1}\right]=\ldots 1010101 \frac{0}{1} \cdot 10(01)^{\infty}$ & 1 & \\
\hline$\left[\bar{x}^{2}\right]=\ldots 10101011 \cdot \frac{1}{0} 10(01)^{\infty}$ & 0 & \\
\hline$\left[\bar{x}^{3}\right]=\ldots 101010 \frac{1}{0} 1.0(01)^{\infty}$ & 2 & \\
\hline$\left[\bar{x}^{4}\right]=\ldots 101 \frac{0}{1} 1001 .(01)^{\infty}$ & 5 & 1 \\
\hline$\left[\bar{x}^{5}\right]=\ldots 101110 \frac{0}{1} 1.0(01)^{\infty}$ & 2 & \\
\hline$\left[\bar{x}^{6}\right]=\ldots 10111011 \cdot \frac{0}{1} 10(01)^{\infty}$ & 0 & \\
\hline$\left[\bar{x}^{7}\right]=\ldots 1011101 \frac{1}{0} \cdot 10(01)^{\infty}$ & 1 & \\
\hline$\left[\bar{x}^{8}\right]=\ldots 10111010 \cdot \frac{1}{0} 10(01)^{\infty}$ & 0 & \\
\hline$\left[\bar{x}^{9}\right]=\ldots 10111 \frac{0}{1} 10 .(01)^{\infty}$ & 3 & \\
\hline$\left[\bar{x}^{10}\right]=\ldots 10111110 \cdot \frac{0}{1} 10(01)^{\infty}$ & 0 & \\
\hline$\left[\bar{x}^{11}\right]=\ldots 1011111 \frac{0}{1} \cdot 10(01)^{\infty}$ & 1 & \\
\hline$\left[\bar{x}^{12}\right]=\ldots 10111111 \cdot \frac{1}{0} 10(01)^{\infty}$ & 0 & \\
\hline$\left[\bar{x}^{13}\right]=\ldots 101111 \frac{1}{0} 1.0(01)^{\infty}$ & 2 & \\
\hline$\left[\bar{x}^{14}\right]=\ldots 10111101 \cdot \frac{0}{1} 10(01)^{\infty}$ & 0 & \\
\hline$\left[\bar{x}^{15}\right]=\ldots 1011110 \frac{1}{0} \cdot 10(01)^{\infty}$ & 1 & \\
\hline$\left[\bar{x}^{16}\right]=\ldots 1011 \frac{1}{0} 100 \cdot(10)^{\infty}$ & 4 & 0 \\
\hline
\end{tabular}

Fig. 2. Composant $C$ and its $p$-points

$E_{p}^{+} \cap \operatorname{int} A=\left\{\left[\bar{x}^{0}\right] \prec \cdots \prec\left[\bar{x}^{n}\right]\right\}$ and $E_{q}^{+} \cap \operatorname{int} B=\left\{\left[\bar{y}^{0}\right] \prec \cdots \prec\left[\bar{y}^{m}\right]\right\}$. Then $m=n$ and either $L_{p}\left[\bar{x}^{i}\right]=L_{q}\left[\bar{y}^{i}\right]$ for every $0 \leq i \leq n$, or $L_{p}\left[\bar{x}^{i}\right]=L_{q}\left[\bar{y}^{n-i}\right]$ for every $0 \leq i \leq n$. This holds because there are $\overleftarrow{a}$ and $\overleftarrow{b}$ such that $A \subseteq A_{\bar{a}}^{p K+k+1}$ and $B \subseteq A_{\bar{b}}^{q K+k+1}$.

Lemma 3.2. Let $p \in \mathbb{Z}_{+}$. Let $\left[\bar{x}^{n}\right] \in E_{p}^{+}$be such that $\left[\bar{x}^{n}\right] \neq \bar{c}^{K}$ and $L_{p}\left[\bar{x}^{n}\right] \neq 0$. Let $i, j \in \mathbb{N}$ be the smallest with $L_{p}\left[\bar{x}^{n+i}\right]>L_{p}\left[\bar{x}^{n}\right]$ and $L_{p}\left[\bar{x}^{n-j}\right]$ $>L_{p}\left[\bar{x}^{n}\right]$. Then the arc between the points $\left[\bar{x}^{n-j}\right]$ and $\left[\bar{x}^{n+i}\right]$ is $\left[\bar{x}^{n}\right]$-symmetric and $L_{p}\left[\bar{x}^{n-k}\right]=L_{p}\left[\bar{x}^{n+k}\right]$ for every $k, 0<k<\min \{i, j\}$.

Proof. The arc between the points $\left[\bar{x}^{n-j}\right]$ and $\left[\bar{x}^{n+i}\right]$ is the basic arc $A_{\bar{x}_{-p K-l-1}^{n}}^{p K+l+1}$, where $l=L_{p}\left[\bar{x}^{n}\right]$. By Proposition 2.10 this arc is $\left[\bar{x}^{n}\right]$-symmetric and $L_{p}\left[\bar{x}^{n-k}\right]=L_{p}\left[\bar{x}^{n+k}\right]$ for every $k, 0<k<\min \{i, j\}$.

REMARK 3.3. In the periodic case one also has

$$
L_{p}\left[\bar{x}^{n+i}\right]-L_{p}\left[\bar{x}^{n}\right] \neq 0(\bmod N) \text { and } L_{p}\left[\bar{x}^{n-j}\right]-L_{p}\left[\bar{x}^{n}\right] \neq 0(\bmod N) \text {. }
$$

Lemma 3.4 (Periodic case). Let $p \in \mathbb{Z}_{+}$and $[\bar{x}],[\bar{y}] \in E_{p},[\bar{x}] \neq[\bar{y}]$. If there is $k \in \mathbb{Z}_{+}$such that $L_{p}[\bar{y}]=L_{p}[\bar{x}]+k N$, then there is $[\bar{z}] \in E_{p}$ between $[\bar{x}]$ and $[\bar{y}]$ such that $L_{p}[\bar{z}]>L_{p}[\bar{x}]$. Moreover, if $k \neq 0$, then there is $[\bar{z}] \in E_{p}$ between $[\bar{x}]$ and $[\bar{y}]$ such that $0<L_{p}[\bar{z}]-L_{p}[\bar{x}]<N$. 
Proof. Since $[\bar{x}],[\bar{y}] \in C$, there is $j \in \mathbb{N}$ such that, for any $\bar{u} \in[\bar{x}]$ and $\bar{v} \in[\bar{y}]$, one has $\overleftarrow{u}_{-j N}=\overleftarrow{c}=\overleftarrow{v}_{-j N}$. Let $m=\max \left\{i \in \mathbb{N}: u_{-i} \neq\right.$ $\left.v_{-i}, \forall \bar{u} \in[\bar{x}], \forall \bar{v} \in[\bar{y}]\right\}$. Since $\left[\vec{x}_{-p N}\right]=\left[\vec{y}_{-p N}\right]$, we have $m>p N$. Since $\overleftarrow{x}_{-m-1}=\overleftarrow{y}_{-m-1}$ and $\left|x_{m}-y_{m}\right|=1$, the basic arcs $A_{\bar{x}_{-m}}^{m}$ and $A_{\bar{y}_{-m}}^{m}$ are neighboring. Note that $[\bar{x}] \in A_{\bar{x}_{-m}}^{m}$ and $[\bar{y}] \in A_{\bar{y}_{-m}}^{m}$. Let $[\bar{z}] \in A_{\bar{x}_{-m}}^{m} \cap A_{\bar{y}_{-m}}^{m}$. Then $\overleftarrow{z}_{-m-1}=\overleftarrow{x}_{-m-1}$ and $\left[\vec{z}_{m}\right]=[\vec{c}]$, and thus, $[\bar{z}] \in E_{p}$. From $m>p N$ it follows that $L_{p}[\bar{z}]>L_{p}[\bar{x}]$.

Suppose $k \neq 0$. Let $L_{p}[\bar{x}]=l$ and $L_{p}[\bar{y}]=l+k N$. Then $\left[\vec{y}_{-(p+k) N-l}\right]$ $=[\vec{c}]$ and $\left[\vec{x}_{-p N-l}\right]=\left[\vec{y}_{-p N-l}\right]=[\vec{c}]$. Since $\left[\vec{x}_{-(p+1) N-l}\right] \neq[\vec{c}]$, there is a smallest $m, l<m<l+N$, such that for any $\bar{u} \in[\bar{x}]$ and $\bar{v} \in[\bar{y}]$ one has $u_{-p N-m} \neq v_{-p N-m}$. The basic arcs $A_{\bar{x}_{-p N-m}}^{p N+m}$ and $A_{\bar{z}_{-p N-m}}^{p N+m}$ with $\overleftarrow{z}_{-p N-m-1}=\overleftarrow{x}_{-p N-m-1}$ and $\left|x_{-p N-m}-z_{-p N-m}\right|=1$ are neighboring. Then, for $[\bar{z}] \in A_{\bar{x}_{-p N-m}}^{p N+m} \cap A_{\bar{z}_{-p N-m}}^{p N+m}$, it follows that $0<L_{p}[\bar{z}]-L_{p}[\bar{x}]<N$.

REMARK 3.5. The corresponding statement for the strictly preperiodic case is the following: Let $p \in \mathbb{Z}_{+}$and $[\bar{x}],[\bar{y}] \in E_{p}^{+},[\bar{x}] \neq[\bar{y}]$. If $L_{p}[\bar{x}]=L_{p}[\bar{y}]$, then there is $[\bar{z}] \in E_{p}^{+}$between $[\bar{x}]$ and $[\bar{y}]$ such that $L_{p}[\bar{z}]>L_{p}[\bar{x}]$. Roughly speaking we can say that in the strictly preperiodic case Lemma 3.4 holds for $k=0$. The proof is similar to that in the periodic case.

Let $\left[\bar{x}^{i}\right],\left[\bar{x}^{j}\right],\left[\bar{x}^{k}\right] \in E_{p}$. If $|i-j|<|i-k|$, the $p$-point $\left[\bar{x}^{i}\right]$ is closer to the $p$-point $\left[\bar{x}^{j}\right]$ than to the $p$-point $\left[\bar{x}^{k}\right]$.

Lemma 3.6 (Periodic case). Let $p \in \mathbb{Z}_{+}, n \in \mathbb{N}$ and $\left[\bar{x}^{n}\right] \in E_{p}$. Let $\left[\bar{x}^{m}\right]$ be the p-point closest to the point $\left[\bar{x}^{n}\right]$ such that $L_{p}\left[\bar{x}^{m}\right]>L_{p}\left[\bar{x}^{n}\right]$. If either $L_{p}\left[\bar{x}^{n}\right]>L_{p}\left[\bar{x}^{m}\right](\bmod N)$, or $L_{p}\left[\bar{x}^{n}\right]>N$, then $L_{p}\left[\bar{x}^{2 n-m}\right]=L_{p}\left[\bar{x}^{m}\right]$ $(\bmod N)$ and $0<L_{p}\left[\bar{x}^{n}\right]-L_{p}\left[\bar{x}^{2 n-m}\right]<N$.

Proof. Let $L_{p}\left[\bar{x}^{n}\right]=r N+l$, for some $r, l \in \mathbb{Z}_{+}, l<N$. Let $i \in \mathbb{N}$ be the smallest number such that $L_{p}\left[\bar{x}^{n+i}\right]>r N+l$, and let $j \in \mathbb{N}$ be the smallest number such that $L_{p}\left[\bar{x}^{n-j}\right]>r N+l$. The arc $A$ between $\left[\bar{x}^{n-j}\right]$ and $\left[\bar{x}^{n+i}\right]$ is the basic arc $A_{\bar{x}_{-(p+r) N-l-1}^{n}}^{(p+r) N+l+1}$. Suppose that $j \leq i$. Then $m=n-j$ and $L_{p}\left[\bar{x}^{m}\right]=k(\bmod N)$. Let $L_{p}\left[\bar{x}^{m}\right]>L_{p}\left[\bar{x}^{n}\right]$. From Lemma 3.2 it follows that the arc $A$ is $\left[\bar{x}^{n}\right]$-symmetric. Therefore, there is $[\bar{z}] \in A,\left[\bar{x}^{n}\right] \prec[\bar{z}]$, such that $\bar{z}_{-(p+r) N-l-1}=\overleftarrow{x}_{-(p+r) N-l-1}^{n}$, $\left[\vec{z}_{-(p+r) N-l+1}\right]=\left[\vec{x}_{-(p+r) N-l+1}^{m}\right]$ and $z_{-(p+r) N-l} \neq x_{-(p+r) N-l}^{m}$. Since $\left[\bar{x}^{m}\right] \in$ $E_{p}, L_{p}\left[\bar{x}^{m}\right]>L_{p}\left[\bar{x}^{n}\right]$ and $k<l$ (respectively $L_{p}\left[\bar{x}^{n}\right]>N$ ), we see that $[\bar{z}] \in E_{p}, r N<L_{p}[\bar{z}] \leq r N+l$ (respectively $r N-N+l<L_{p}[\bar{z}] \leq r N+l$ ) and $L_{p}[\bar{z}]=k(\bmod N)$. Hence, $[\bar{z}]=\left[\bar{x}^{n+j}\right]=\left[\bar{x}^{2 n-m}\right]$. From Lemma 3.4 it follows that $L_{p}\left[\bar{x}^{n}\right]>L_{p}\left[\bar{x}^{2 n-m}\right]$ and thus $i \neq j$. Lemma 3.2 yields $L_{p}[\bar{z}] \neq r N+l-N$ and $0<L_{p}\left[\bar{x}^{n}\right]-L_{p}\left[\bar{x}^{2 n-m}\right]<N$. In the case $i<j$ the proof is analogous. 
Corollary 3.7 (Periodic case). Let $p \in \mathbb{Z}_{+}$and $\left[\bar{x}^{n}\right],\left[\bar{x}^{m}\right] \in E_{p}$, $|m-n| \geq 2$. If there is $k \in \mathbb{Z}_{+}$such that $L_{p}\left[\bar{x}^{m}\right]=L_{p}\left[\bar{x}^{n}\right]+k N$ and, for every $i \in \mathbb{Z}_{+}$and $n<j<m, L_{p}\left[\bar{x}^{j}\right] \neq L_{p}\left[\bar{x}^{n}\right]+i N$, then $n+m$ is even and, for $l=\max \left\{L_{p}\left[\bar{x}^{j}\right]: n<j<m\right\}$, one has $L_{p}\left[\bar{x}^{n}\right]<l=L_{p}\left[\bar{x}^{(n+m) / 2}\right]$. Moreover, if $k \neq 0$, then $l<L_{p}\left[\bar{x}^{n}\right]+N$.

Proof. From Lemma 3.4 it follows that $L_{p}\left[\bar{x}^{n}\right]<l$ and there is a unique $j, n<j<m$, with $L_{p}\left[\bar{x}^{j}\right]=l$. The condition $j-n<m-j$ is not possible, because from $L_{p}\left[\bar{x}^{n}\right]<l$ and from Lemma 3.2 it follows that $L_{p}\left[\bar{x}^{2 j-n}\right]=$ $L_{p}\left[\bar{x}^{n}\right]$, which contradicts the assumptions of the corollary. Suppose that $j-n>m-j$. If $L_{p}\left[\bar{x}^{m}\right]<l$, then $L_{p}\left[\bar{x}^{2 j-m}\right]=L_{p}\left[\bar{x}^{m}\right]$, which contradicts the assumptions of the corollary again. If $L_{p}\left[\bar{x}^{m}\right]>l$, the conditions of Lemma 3.6 are satisfied, since $l>L_{p}\left[\bar{x}^{n}\right]$. Hence, $L_{p}\left[\bar{x}^{2 j-m}\right]=L_{p}\left[\bar{x}^{n}\right]+i N$ for some $i \in \mathbb{Z}_{+}$, which again contradicts the assumptions of the corollary. Therefore, $n+m$ is even and $j=(n+m) / 2$. Now, if $k \neq 0$, it follows from Lemma 3.6 that $l<L_{p}\left[\bar{x}^{n}\right]+N$.

REMARK 3.8. In the strictly preperiodic case, Corollary 3.7 holds for $k=0$. The proof is similar to that in the periodic case.

Lemma 3.9 (Periodic case). Let $p \in \mathbb{Z}_{+}$. Let $[\bar{x}],[\bar{y}] \in E_{p}$ be such that $L_{p}[\bar{x}]=i N+k, L_{p}[\bar{y}]=j N+k+1, i, j, k \in \mathbb{Z}_{+}, k<N$, and there is no $[\bar{w}] \in E_{p}$ between $[\bar{x}]$ and $[\bar{y}]$ satisfying $L_{p}[\bar{w}] \geq \min \{i, j\} N+k$ and either $L_{p}[\bar{w}]=k(\bmod N)$ or $L_{p}[\bar{w}]=k+1(\bmod N)$. Then, for any $n<$ $\min \{i, j\} N+k$, there is $[\bar{z}] \in E_{p}$ between $[\bar{x}]$ and $[\bar{y}]$, such that $L_{p}[\bar{z}]=n$.

Proof. It is sufficient to prove the statement for $n=\min \{i, j\} N+k-1$. Let $m=\min \{i, j\}$. Without loss of generality we can suppose that $[\bar{x}] \prec[\bar{y}]$.

First, let us show that there is no $[\bar{w}] \in E_{p},[\bar{x}] \prec[\bar{w}] \prec[\bar{y}]$, such that $L_{p}[\bar{w}]>m N+k$. Because of Lemma 3.2 , there is no $[\bar{w}] \in E_{p},[\bar{x}] \prec[\bar{w}] \prec$ $[\bar{y}]$, such that $L_{p}[\bar{w}]>\max \left\{L_{p}[\bar{x}], L_{p}[\bar{y}]\right\}$. Suppose that $L_{p}[\bar{x}]<L_{p}[\bar{y}]$ and there is $[\bar{w}] \in E_{p}$ such that $L_{p}[\bar{x}]<L_{p}[\bar{w}]<L_{p}[\bar{y}]$. By Lemma 3.2, the point $[\bar{w}]$ is closer to $[\bar{y}]$ than to $[\bar{x}]$. By Lemma 3.6, one concludes that $L_{p}[\bar{w}]<k$, which contradicts the assumption that $L_{p}[\bar{x}]<L_{p}[\bar{w}]$. Under the assumption that $L_{p}[\bar{y}]<L_{p}[\bar{w}]<L_{p}[\bar{x}]$, the conclusion can be obtained analogously. Therefore, for every $[\bar{w}] \in E_{p},[\bar{x}] \prec[\bar{w}] \prec[\bar{y}]$, one concludes that $L_{p}[\bar{w}]<m N+k$. Hence, $\left\{[\vec{v}]: \vec{v}=\vec{w}_{-p N-m N-k+1},[\bar{x}] \preceq[\bar{w}] \preceq[\bar{y}]\right\}$ is homeomorphic to a closed interval. Since $\left[\vec{x}_{-p N-m N-k+1}\right]=\left[\vec{c}_{1}\right]$ and $\left[\vec{y}_{-p N-m N-k+1}\right]=\left[\vec{c}_{2}\right]$, this closed interval is $[0,1]$ and there is $[\bar{z}] \in E_{p}$, $[\bar{x}] \prec[\bar{z}] \prec[\bar{y}]$, such that $L_{p}[\bar{z}]=m N+k-1$.

REMARK 3.10. In the strictly preperiodic case, Lemma 3.9 holds for $i=j=0$. The proof is similar to that in the periodic case.

An arc $A$ of the composant $C$ such that $\partial A \in E_{p}$ and $A \cap E_{p}=$ $\left\{\left[\bar{y}^{0}\right], \ldots,\left[\bar{y}^{n}\right]\right\}$ is called p-symmetric if $\left[\vec{y}_{-p N}^{0}\right]=\left[\vec{y}_{-p N}^{n}\right]$ and $L_{p}\left[\bar{y}^{i}\right]=$ 


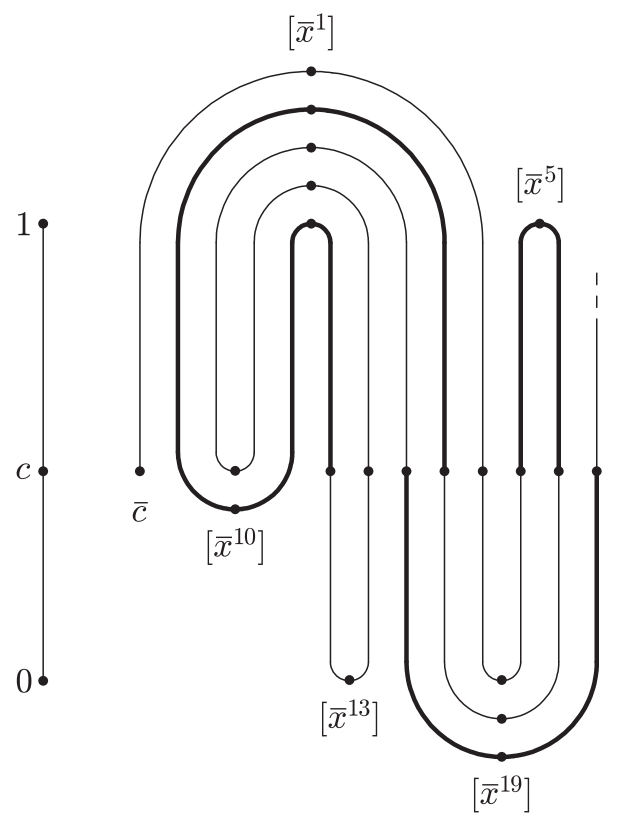

\begin{tabular}{|c|c|c|}
\hline$E_{0}$ & $L_{0}$ & $L_{1}$ \\
\hline $\bar{c}=\ldots 110110 .(110)^{\infty}$ & $\infty$ & $\infty$ \\
\hline$\left[\bar{x}^{1}\right]=\ldots 11011 \frac{0}{1} \cdot(101)^{\infty}$ & 1 & \\
\hline$\left[\bar{x}^{2}\right]=\ldots 110111 \cdot \frac{1}{0}(101)^{\infty}$ & 0 & \\
\hline$\left[\bar{x}^{3}\right]=\ldots 1101 \frac{1}{0} 1 .(011)^{\infty}$ & 2 & \\
\hline$\left[\bar{x}^{4}\right]=\ldots 110101 \cdot \frac{0}{1}(101)^{\infty}$ & $\mathbf{0}$ & \\
\hline$\left[\bar{x}^{5}\right]=\ldots 11 \frac{0}{1} 101 .(101)^{\infty}$ & 4 & 1 \\
\hline$\left[\bar{x}^{6}\right]=\ldots 111101 \cdot \frac{1}{0}(101)^{\infty}$ & $\mathbf{0}$ & \\
\hline$\left[\bar{x}^{7}\right]=\ldots 1111 \frac{0}{1} 1 .(011)^{\infty}$ & 2 & \\
\hline$\left[\bar{x}^{8}\right]=\ldots 111111 \cdot \frac{0}{1}(101)^{\infty}$ & 0 & \\
\hline$\left[\bar{x}^{9}\right]=\ldots 11111 \frac{1}{0} \cdot(101)^{\infty}$ & 1 & \\
\hline$\left[\bar{x}^{10}\right]=\ldots 111 \frac{1}{0} 10 \cdot(110)^{\infty}$ & 3 & 0 \\
\hline$\left[\bar{x}^{11}\right]=\ldots 11101 \frac{0}{1} \cdot(101)^{\infty}$ & 1 & \\
\hline$\left[\bar{x}^{12}\right]=\ldots 111011 \cdot \frac{1}{0}(101)^{\infty}$ & $\mathbf{0}$ & \\
\hline$\left[\bar{x}^{13}\right]=\ldots 1 \frac{1}{0} 1011 .(011)^{\infty}$ & 5 & 2 \\
\hline$\left[\bar{x}^{14}\right]=\ldots 101011 \cdot \frac{0}{1}(101)^{\infty}$ & 0 & \\
\hline$\left[\bar{x}^{15}\right]=\ldots 10101 \frac{1}{0} \cdot(101)^{\infty}$ & 1 & \\
\hline$\left[\bar{x}^{16}\right]=\ldots 101 \frac{0}{1} 10 \cdot(110)^{\infty}$ & 3 & 0 \\
\hline
\end{tabular}

Fig. 3. Composant $C$ and its $p$-bridges

$L_{p}\left[\bar{y}^{n-i}\right]$ for every $0<i<n$. Every $q$-symmetric arc is also $p$-symmetric for every $0 \leq p \leq q$. Note that if $A$ is a $p$-symmetric arc of $C$ and $A \cap E_{p}=$ $\left\{\left[\bar{x}^{0}\right], \ldots,\left[\bar{x}^{n}\right]\right\}$, then by Lemma $3.4, n$ is even. The $p$-point $\left[\bar{x}^{n / 2}\right]$ is called the center of $A$, it is denoted by $\left[\bar{\chi}^{A}\right]$, and $L_{p}\left[\bar{\chi}^{A}\right]=\max \left\{L_{p}[\bar{x}]:[\bar{x}] \in\right.$ $\left.E_{p} \cap \operatorname{int} A\right\}$. Therefore, the centers of the $p$-symmetric arcs of the composant $C$ are the "turning points" of $C$. In order to describe the folding pattern of $C$, let us define some special arcs.

Definition 3.11. Let $p \in \mathbb{Z}_{+}$. An arc $B$ of the composant $C$ is called a p-bridge if $\partial B \subset E_{p}, L_{p}[\bar{x}]=0$ for every $[\bar{x}] \in \partial B$, and $L_{p}[\bar{x}] \neq 0$ for every $[\bar{x}] \in \operatorname{int} B$.

In Figure 3 some 0-bridges of $C$ from Example 3.1, the periodic case, are marked. From Corollary 3.7 and Lemma 3.2 it is easy to see that every $p$-bridge is $p$-symmetric.

For $p \in \mathbb{Z}_{+}$, let $B$ be a $p$-bridge of $C$. Let $B \cap E_{p}=\left\{\left[\bar{x}^{0}\right], \ldots,\left[\bar{x}^{n}\right]\right\}$. For $q \leq p$, let $B \cap E_{q}=\left\{\left[\bar{z}^{0}\right], \ldots,\left[\bar{z}^{m}\right]\right\}$. We will call the finite sequence $L_{q}\left[\bar{z}^{1}\right], \ldots, L_{q}\left[\bar{z}^{m-1}\right]$ the $q$-folding pattern of the $p$-bridge $B$. Let $k \in\{1, \ldots$ $\ldots, n-1\}$ be such that $L_{p}\left[\bar{x}^{i}\right] \neq L_{p}\left[\bar{x}^{k}\right]$ for every $i \in\{1, \ldots, k-1\}$. Then, by Lemma 3.2, one has $L_{p}\left[\bar{x}^{i}\right]<L_{p}\left[\bar{x}^{k}\right]$ for every $i \in\{1, \ldots, k-1\}$. For $q \in \mathbb{Z}_{+}$ 
let $D$ be a $q$-bridge of $C$. Let $D \cap E_{q}=\left\{\left[\bar{y}^{0}\right], \ldots,\left[\bar{y}^{m}\right]\right\}$. If $L_{p}\left[\bar{\chi}^{B}\right]=L_{q}\left[\bar{\chi}^{D}\right]$ $=l$, then $l=\max \left\{L_{p}[\bar{x}]:[\bar{x}] \in E_{p} \cap \operatorname{int} B\right\}=\max \left\{L_{q}[\bar{x}]:[\bar{x}] \in E_{q} \cap \operatorname{int} D\right\}$, $\left[\vec{\chi}_{-p N-l}^{B}\right]=[\vec{c}]=\left[\vec{\chi}_{-q N-l}^{D}\right]$ and $\pi_{p N+l}(B)=\pi_{q N+l}(D)$. Therefore, $m=n$ and $L_{p}\left[\bar{x}^{i}\right]=L_{q}\left[\bar{y}^{i}\right]$ for every $0<i<n$. Hence, $L_{p}\left[\bar{\chi}^{B}\right]$ determines the $q$-folding pattern of the $p$-bridge $B$ for all $q \leq p$. Consequently, it is natural to ask which kinds of $p$-bridges with respect to the $p$-levels of their centers exist.

Lemma 3.12. Let $p \in \mathbb{Z}_{+}$and $n \in \mathbb{N}$. There is a p-bridge $B \subset C$ such that $L_{p}\left[\bar{\chi}^{B}\right]=n$ if and only if $c_{s} \in f_{s}^{n}\left(\left[0, c_{s}\right]\right)$.

Proof. Let $B$ be a $p$-bridge such that $L_{p}\left[\bar{\chi}^{B}\right]=n$. Then $\overleftarrow{x}_{-p N-n-1}=$ $\overleftarrow{\chi}_{-p N-n-1}^{B}$ for every $[\bar{x}] \in B$, and $\left|y_{-p N-n}-z_{-p N-n}\right|=1$ for $[\bar{y}],[\bar{z}] \in \partial B$, $[\bar{y}] \neq[\bar{z}]$. Without loss of generality we can suppose that $y_{-p N-n}=0$. Since $[\bar{y}] \in \partial B$, we have $\left[\vec{y}_{-p N}\right]=[\vec{c}]$, and thus, $\widetilde{\sigma}^{n}\left[\vec{y}_{-p N-n}\right]=[\vec{c}]$. Therefore, there is $\xi \in\left[0, c_{s}\right]$ such that $f_{s}^{n}(\xi)=c_{s}$.

Let $\overleftarrow{a}$ be the sequence which describes the composant $C$ such that $\overleftarrow{a} \vec{c}_{1}$ and $\overleftarrow{a}_{c_{2}}$ are allowed. Then the basic arc $A_{\bar{a}}^{p N+n+1}$ contains a point $[\bar{z}]$ such that $\left[\vec{z}_{-p N-n}\right]=[\vec{c}]$. Let $c_{s} \in f_{s}^{n}\left(\left[0, c_{s}\right]\right)$. Then there is a $p$-point $[\bar{x}] \in$ $A_{\bar{a}}^{p N+n+1}$ with $x_{-p N-n}=0, L_{p}[\bar{x}]=0$, and there is no point $[\bar{u}] \in A_{\bar{a}}^{p N+n+1}$, $[\bar{x}] \prec[\bar{u}] \prec[\bar{z}]$, with $u_{-p N-n}=0, L_{p}[\bar{u}]=0$. By Proposition 2.10, the basic arc $A_{\bar{a}}^{p N+n+1}$ is $[\bar{z}]$-symmetric and there is $[\bar{y}] \in A_{\bar{a}}^{p N+n+1}$ such that $y_{-p N-n}=1$ and $\left[\vec{y}_{-p N-n+1}\right]=\left[\vec{x}_{-p N-n+1}\right]$. Then the arc between the $p$-points $[\bar{x}]$ and $[\bar{y}]$ is the required $p$-bridge $B,\left[\bar{\chi}^{B}\right]=[\bar{z}]$ and $L_{p}\left[\bar{\chi}^{B}\right]=n$.

Corollary 3.13. Let $p \in \mathbb{Z}_{+}$. If $c^{3} \leq c_{s}$, then for every $n \in \mathbb{N}$, there is a p-bridge $B \subset C$ such that $L_{p}\left[\bar{\chi}^{B}\right]=n$.

Proof. If $c^{3} \leq c_{s}$, then $c_{s} \in f_{s}\left(\left[0, c_{s}\right]\right)=\left[c^{3}, c^{1}\right]$ and $f_{s}^{2}\left(\left[0, c_{s}\right]\right)=I$.

Lemma 3.14. Let $p \in \mathbb{Z}_{+}$. For every $n \in \mathbb{N}$, there is a p-bridge $B \subset C$ such that $L_{p}\left[\bar{\chi}^{B}\right]=2 n$.

Proof. If $c^{3} \leq c_{s}$ the statement follows from Corollary 3.13. Let $c^{3}>c_{s}$. Then $c^{4} \geq c_{s}$. If this were not valid, i.e., $c^{4}<c_{s}$, then $f_{s}\left(\left(0, c_{s}\right)\right)=\left(c^{3}, 1\right)$ and $f_{s}\left(\left(c^{3}, 1\right)\right)=\left(0, c^{4}\right) \subset\left(0, c_{s}\right)$, contradicting the assumption that $f_{s}$ is l.e.o. Therefore $\left[0, c_{s}\right] \subseteq f_{s}^{2}\left(\left[0, c_{s}\right]\right)=\left[0, c^{4}\right]$. We deduce, by induction, that $\left[0, c_{s}\right] \subseteq f_{s}^{2(n-1)}\left(\left[0, c_{s}\right]\right) \subseteq f_{s}^{2 n}\left(\left[0, c_{s}\right]\right)$ for every $n \in \mathbb{N}$, and the statement follows from Lemma 3.12 .

Lemma 3.15. Let $p \in \mathbb{Z}_{+}$and $m=\min \left\{i \in \mathbb{N}: f_{s}^{2 i+1}\left(c_{s}\right) \in\left[0, c_{s}\right]\right\}$. There is a p-bridge $B \subset C$ such that $L_{p}\left[\bar{\chi}^{B}\right]=2 n-1$ if and only if $n \geq m$.

Proof. Let us first show that there is $j \in \mathbb{N}$ with $f_{s}^{2 j+1}\left(c_{s}\right) \in\left[0, c_{s}\right]$. If $c^{3} \leq c_{s}$ then $j=1$. Let $c^{3}>c_{s}$. Then $c^{4}>c_{s}$. Let us show that $c^{3}<c^{4}$. Assume that, on the contrary, $c^{4}<c^{3}$. Since $\left.f_{s}\right|_{\left[c_{s}, 1\right]}$ is strictly decreasing, 
one obtains $f_{s}\left(c^{4}\right)=c^{5}>c^{4}=f_{s}\left(c^{3}\right)$. From $s>1$ it follows that $c^{3}-c^{4}<$ $c^{5}-c^{4}$, implying $c^{3}<c^{5}$. But then $f_{s}\left(\left[0, c^{4}\right]\right)=\left[c^{3}, 1\right]$ and $f_{s}^{2 i+1}\left(\left[0, c^{4}\right]\right)=$ $\left[c^{3}, 1\right]$ for every $i \in \mathbb{N}$, which is impossible, because $f_{s}$ is l.e.o. Therefore, $c^{3}<c^{4}$ and $c^{4}>c^{5}$. From $c^{4}-c^{3}<c^{4}-c^{5}$, it follows that $c^{3}>c^{5}$. If $c^{5}>c_{s}$, then $c^{4}<c^{6}$. Hence, for every $i \in \mathbb{N}, c_{s}<c^{2 i+1}$ implies

$$
c^{2 i+3}<c^{2 i+1} \leq c^{3}<c^{4} \leq c^{2 i+2} .
$$

Since $f_{s}$ is l.e.o., there is $j \in \mathbb{N}$ such that $f_{s}^{2 j+1}\left(c_{s}\right) \in\left[0, c_{s}\right]$.

Let $m \in \mathbb{N}$ be the smallest such that $f_{s}^{2 m+1}\left(c_{s}\right) \in\left[0, c_{s}\right]$. If $m=1$ the statement of the lemma follows from Corollary 3.13. Let $m>1$. Since for every $n \in \mathbb{N},\left[0, c_{s}\right] \subseteq f_{s}^{2 n}\left(\left[0, c_{s}\right]\right)$, one concludes that $\left[c^{3}, 1\right] \subseteq f_{s}^{2 n+1}\left(\left[0, c_{s}\right]\right)$. Hence, it follows by $(1)$ that $f_{s}^{2 n-1}\left(\left[0, c_{s}\right]\right)=\left[c^{2 n+1}, 1\right]$ for every $n<m$. Therefore, $c_{s} \notin f_{s}^{2 n+1}\left(\left[0, c_{s}\right]\right)$ for every $n<m$, and $c_{s} \in f_{s}^{2 n+1}\left(\left[0, c_{s}\right]\right)$ for every $n \geq m$. Now, the statement follows from Lemma 3.12.

Corollary 3.16. Let $p \in \mathbb{Z}_{+}$. For every $n \in \mathbb{N}, n \geq M+N-2$, there is a p-bridge $B \subset C$ such that $L_{p}\left[\bar{\chi}^{B}\right]=n$. Also, for every $j \in\{M, \ldots, M+$ $N-1\}$, there is a p-bridge $B \subset C$ such that $\vec{\chi}_{-p N}^{B}=\vec{c}_{j}$.

Corollary 3.17 (Periodic case). Let $p \in \mathbb{Z}_{+}$and let $[\bar{x}],[\bar{y}] \in E_{p}$ be such that $L_{p}[\bar{x}]=i N+k, L_{p}[\bar{y}]=j N+k+1, i, j, k \in \mathbb{Z}_{+}, k<N$. Then for every $n<\min \{i, j\} N+k$, there is $[\bar{z}] \in E_{p}$ between $[\bar{x}]$ and $[\bar{y}]$ such that $L_{p}[\bar{z}]=n$. Furthermore, either there is a p-bridge $B$ between $[\bar{x}]$ and $[\bar{y}]$ such that $L_{p}\left[\bar{\chi}^{B}\right]=n$, or there is no $p$-bridge whose center has $p$-level $n$.

Proof. It is sufficient to prove the statement for $n=\min \{i, j\} N+k-1$. Let $m=\min \{i, j\}$. Without loss of generality we can assume that $[\bar{x}] \prec[\bar{y}]$. Let $[\bar{u}]$ be the $p$-point closest to $[\bar{y}]$ such that $[\bar{u}] \prec[\bar{y}], L_{p}[\bar{u}] \geq m N+k$ and $L_{p}[\bar{u}]=k(\bmod N)$. Let $[\bar{v}]$ be the $p$-point closest to $[\bar{u}]$ such that $[\bar{u}] \prec[\bar{v}], L_{p}[\bar{v}] \geq m N+k+1$ and $L_{p}[\bar{v}]=k+1(\bmod N)$. We assert that at least one of the inequalities $L_{p}[\bar{u}] \geq m N+k, L_{p}[\bar{v}] \geq m N+k+1$ is an equality. Suppose not, i.e. $L_{p}[\bar{u}] \geq(m+1) N+k$ and $L_{p}[\bar{v}] \geq(m+1) N+$ $k+1$. Then, by Lemma 3.9, there is $[\bar{z}] \in E_{p},[\bar{u}] \prec[\bar{z}] \prec[\bar{v}]$, such that $L_{p}[\bar{z}]=m N+k+1$, contradicting the choice of $[\bar{v}]$. Now the first statement follows from Lemma 3.9, and the second from the proof of Lemma 3.12 with $\overleftarrow{a}=\overleftarrow{w}_{-p N-m N-k}$ for some $[\bar{u}] \prec[\bar{w}] \prec[\bar{v}]$

REMARK 3.18. In the strictly preperiodic case, Corollary 3.17 holds for $i=j=0$. The proof is similar to that in the periodic case.

Let $p \in \mathbb{Z}_{+}$, let $B \subset C$ be a $p$-bridge and $B \cap E_{p}=\left\{\left[\bar{x}^{0}\right], \ldots,\left[\bar{x}^{n}\right]\right\}$. Let $T(B)=\min \left\{L_{p}\left[\bar{\chi}^{A}\right]: A\right.$ is a $p$-bridge, $A \cap E_{p}=\left\{\left[\bar{u}^{0}\right], \ldots,\left[\bar{u}^{n}\right]\right\},\left[\vec{u}_{-p N}^{i}\right]=$ $\left.\left[\vec{x}_{-p N}^{i}\right], 0 \leq i \leq n\right\}$. Let $q \in \mathbb{Z}_{+}$, let $D \subset C$ be a $q$-bridge and $D \cap E_{q}=$ 
$\left\{\left[\bar{y}^{0}\right], \ldots,\left[\bar{y}^{m}\right]\right\}$. If $T(B)=T(D)$, then there are a $p$-bridge $B_{1} \subset C$ and a $q$ bridge $D_{1} \subset C$ with $L_{p}\left[\bar{\chi}^{B_{1}}\right]=L_{q}\left[\bar{\chi}^{D_{1}}\right]$. Hence, $m=n$ and $\left[\vec{x}_{-p N}^{i}\right]=\left[\vec{y}_{-q N}^{i}\right]$ for every $0 \leq i \leq n$. Therefore, we will call the number $T(B)$ the type of the $p$-bridge $B$. Moreover, the following lemma is valid.

Lemma 3.19. Let $p, q \in \mathbb{Z}_{+}$. Let $B \subset C$ be a p-bridge and $D \subset C$ be a q-bridge. Let $B \cap E_{p}=\left\{\left[\bar{x}^{0}\right], \ldots,\left[\bar{x}^{n}\right]\right\}, D \cap E_{q}=\left\{\left[\bar{y}^{0}\right], \ldots,\left[\bar{y}^{m}\right]\right\}$. If $T(B)=T(D)$, then $m=n$ and $L_{p}\left[\bar{x}^{i}\right]=L_{q}\left[\bar{y}^{i}\right]$ for every $0 \leq i \leq n, i \neq n / 2$.

Proof. Since $T(B)=T(D)$, we have $m=n$. Without loss of generality we can assume that $L_{p}\left[\bar{\chi}^{B}\right]=T(B)$. Let $\left[\bar{y}^{k}\right]$ be the first $q$-point of $D$ such that $L_{q}\left[\bar{y}^{k}\right] \geq T(B)$ and $\left[\vec{y}_{-q N}^{k}\right]=\left[\vec{\chi}_{-q N}^{D}\right]$. Such a $k$ exists since $\left[\bar{\chi}^{D}\right]$ satisfies these conditions. Since $\left[\vec{x}_{-p K}^{k}\right]=\left[\vec{y}_{-q K}^{k}\right]$ and $L_{p}\left[\bar{x}^{k}\right]<T(B)$, there is $j_{1} \in \mathbb{N}$ with $L_{q}\left[\bar{y}^{k}\right]=L_{p}\left[\bar{x}^{k}\right]+j_{1} K$. Let $D_{k}$ be the arc between the points $\left[\bar{y}^{0}\right]$ and $\left[\bar{y}^{k}\right]$. Let us first show that

$$
\overleftarrow{y}_{-q N-T(B)}=\overleftarrow{z}_{-q N-T(B)}
$$

for any two points $[\bar{y}],[\bar{z}] \in \operatorname{int} D_{k}$. If $k=1$, then $D_{1}$ is a basic arc and the statement holds. Let $k>1$, and let $l \in \mathbb{N}$ be the largest number such that $L_{q}\left[\bar{y}^{l}\right]=\max \left\{L_{q}\left[\bar{y}^{i}\right]: 0<i<k\right\}$. If $L_{q}\left[\bar{y}^{l}\right] \geq T(B)$, since $\left[\vec{x}_{-p K}^{l}\right]=\left[\vec{y}_{-q K}^{l}\right]$ and $L_{p}\left[\bar{x}^{l}\right]<T(B)$, there is $j_{2} \in \mathbb{N}$ with $L_{q}\left[\bar{y}^{l}\right]=L_{p}\left[\bar{x}^{l}\right]+j_{2} K$. Then $\operatorname{card}\left\{[\bar{y}] \in E_{q}:\left[\bar{y}^{l}\right] \prec[\bar{y}] \prec\left[\bar{y}^{k}\right]\right\}>\operatorname{card}\left\{[\bar{x}] \in E_{p}:\left[\bar{x}^{l}\right] \prec[\bar{x}] \prec\left[\bar{x}^{k}\right]\right\}$, which is impossible because $m=n$. Therefore, (2) holds. Since $L_{p}\left[\bar{\chi}^{B}\right] \geq L_{p}\left[\bar{x}^{i}\right]$ for every $0<i<n, \overleftarrow{x}_{-p N-T(B)-1}=\overleftarrow{\chi}_{-p N-T(B)-1}^{B}$ for every $[\bar{x}] \in \operatorname{int} B$. Let $B_{1}$ be the arc between the points $\left[\bar{x}^{0}\right]$ and $\left[\bar{x}^{n / 2}\right]$. Since every $p$-bridge is $p$-symmetric, it follows from (2) that $\pi_{q N+T(B)}\left(D_{k}\right)=\pi_{p N+T(B)}\left(B_{1}\right)$. Hence, $L_{p}\left[\bar{x}^{i}\right]=L_{q}\left[\bar{y}^{i}\right]$ for every $0<i<n, i \neq n / 2$.

THEOREM 3.20. There are finitely many bridge types.

Proof. Suppose that, on the contrary, there are infinitely many bridge types. Since for every $[\bar{x}] \in E_{p}$ one has $\vec{x}_{-p K} \in\left\{\vec{c}_{i}: i \in\{0, \ldots, M+N-1\}\right\}$, it follows that for every $i \in \mathbb{N}$ there exists a $p$-bridge $B^{i}$ which contains a $p$-point $\left[\bar{x}^{m_{i}}\right],\left[\bar{x}^{m_{i}}\right] \neq\left[\bar{\chi}^{B^{i}}\right]$, such that $L_{p}\left[\bar{x}^{m_{i}}\right]=n_{i}=\max \left\{L_{p}[\bar{x}]:[\bar{x}] \in\right.$ $\left.B^{i} \cap E_{p},[\bar{x}] \neq\left[\bar{\chi}^{B^{i}}\right]\right\}$ and the sequence $\left(n_{i}\right)_{i \in \mathbb{N}}$ is strictly increasing. Also, for every such $p$-bridge $B^{i}$, there exists a $p$-bridge $A$ with the following properties:

(a) the $p$-bridge $A$ contains only three $p$-points and $L_{p}\left[\bar{\chi}^{A}\right]=n$ for some $n \in \mathbb{N}$ (both neighboring $p$-points of $\left[\bar{\chi}^{A}\right]$ have $p$-level 0 ),

(b) $\widetilde{\sigma}^{n_{i}}(A)=A^{n_{i}} \subset B^{i}$,

(c) $\left[\bar{x}^{m_{i}}\right] \in \partial A^{n_{i}}$.

Note that $L_{p}\left[\bar{x}^{m_{i}}\right]=n_{i}$ and $n=L_{p}\left[\bar{\chi}^{B^{i}}\right]-n_{i}$ (in the periodic case $n \neq j N$ for every $j \in \mathbb{N}$ ). Fix some $p$-bridge $B^{l} \in\left(B^{i}\right)_{i \in \mathbb{N}}$ and the corresponding 
$p$-bridge $A$. We will study the $\operatorname{arcs} A^{i}=\widetilde{\sigma}^{i}(A), i \leq n_{l}$. By (a) and (b), one has $c_{n+1}=c_{1}$ (otherwise $A^{1}$ contains $p$-points of $p$-levels 1 and 0 implying $\left.A^{n_{l}} \nsubseteq B^{l}\right)$. Note that the only properties of $n$ we used are the following:

(i) $n$ is the $p$-level of a $p$-point both of whose neighboring $p$-points have $p$-level 0 ,

(ii) there is a $p$-bridge $A$ whose center has $p$-level $n$, such that $\widetilde{\sigma}^{k_{l}}(A)=$ $A^{k_{l}} \subset B^{l}$ for some $k_{l} \in \mathbb{N}$.

Suppose that we have proved that there exists $j \in \mathbb{N}$ such that, for every $n$ which satisfies (i) and (ii), one has $c_{i}=c_{n+i}$ for every $i<j$. Suppose that $c_{j} \neq c_{n+j}$. Thus, $c_{n+j+1}=c_{j+1}=c_{1}$ (otherwise $A^{j+1}$ contains $p$-points of $p$-levels 1 and 0 implying $A^{n_{l}} \nsubseteq B^{l}$ ). Since $j$ satisfies (i) and (ii), one has $c_{j+i}=c_{i}$ for every $i<j$. Therefore, $c_{k j+i}=c_{i}$ for every $i<j$ and for every $k$ such that $(k+1) j<n_{l}$. Since the sequence $\left(n_{i}\right)_{i \in \mathbb{N}}$ is strictly increasing, this implies that $\vec{c}_{1}=10 c_{3} \ldots c_{j-1} * \vec{y}$ for some $\vec{y}$, where $*$ denotes the $*$-product defined in [C-E, p. 72]. Hence it follows that there is a closed interval $J$ with $f_{s}^{j}(J) \subset J$ ([C-E, pp. 72-73]), which is impossible since $f_{s}$ is l.e.o. Therefore, $c_{i}=c_{n+i}$ for every $i$. Then, in the strictly preperiodic case, the preperiod of $\left[\vec{c}_{1}\right]$ would be less than $M$, contrary to assumption. In the periodic case, since $n \neq j N$ for every $j \in \mathbb{N}$, this contradicts the assumption that $N$ is the period of $\left[\vec{c}_{1}\right]$. The contradictions established in the last two sentences show that it cannot be the case that $c_{i}=c_{n+i}$ for every $i$. Hence, the sequence $\left(n_{i}\right)_{i \in \mathbb{N}}$ cannot be strictly increasing. The derived contradiction implies that there are finitely many bridge types.

We will now consider relations between different bridges of the composant $C$. For two $p$-bridges $B^{1}, B^{2} \subset C$, we say that $B^{1} \prec B^{2}$ if for every $[\bar{x}] \in B^{1}$ and every $[\bar{y}] \in B^{2}$, one has $[\bar{x}] \preceq[\bar{y}]$. Let $B \subset C$ be a $p$-bridge and $B \cap E_{p-1}=\left\{\left[\bar{x}^{0}\right], \ldots,\left[\bar{x}^{n}\right]\right\}$. The arc between $\left[\bar{x}^{0}\right]$ and $\left[\bar{\chi}^{B}\right]$ will be denoted by $A^{2}$, and the arc between $\left[\bar{\chi}^{B}\right]$ and $\left[\bar{x}^{n}\right]$ by $A^{1}$. The arcs $A^{1}$ and $A^{2}$ will be called the $(p-1)$-semibridges. Note that $L_{p-1}\left[\bar{x}^{i}\right]=L_{p-1}\left[\bar{x}^{n-i}\right]$ for every $i \in$ $\{0, \ldots, n / 2\}$. We say that the $(p-1)$-semibridges $A^{1}$ and $A^{2}$ have the semitype $s T\left(A^{1}\right)=s T\left(A^{2}\right)=T(B)$. Let $A$ be an arc such that $\partial A \subset E_{p-1}$ and let $A \cap E_{p-1}=\left\{\left[\bar{y}^{0}\right], \ldots,\left[\bar{y}^{m}\right]\right\}$. If $m=n / 2$ and either $L_{p-1}\left[\bar{y}^{i}\right]=L_{p-1}\left[\bar{x}^{i}\right]$ for every $i \in\{0, \ldots, n / 2-1\}$ and $\left[\vec{y}_{-(p-1) K}^{m}\right]=\left[\vec{\chi}_{-(p-1) K}^{B}\right]$, or $L_{p-1}\left[\bar{y}^{i}\right]=$ $L_{p-1}\left[\bar{x}^{n / 2+i}\right]$ for every $i \in\{1, \ldots, n / 2\}$ and $\left[\vec{y}_{-(p-1) K}^{0}\right]=\left[\vec{\chi}_{-(p-1) K}^{B}\right]$, then the $\operatorname{arc} A$ is a $(p-1)$-semibridge with semitype $s T(A)=T(B)$.

In the strictly preperiodic case we defined $K=2 k N$ for some $k \in \mathbb{N}$. Let $k$ be the smallest such that, for every $p \in \mathbb{Z}_{+}$, all $p$-bridges whose centers have $p$-level $i K, i \in \mathbb{N}$, have the same type, $K$, and every $p$-bridge which is not of type $K$ does not contain any $p$-point of $p$-level $K$. By Theorem 3.20 such a $k$ exists. Note that in the periodic case, $K=N$ as before. 
Let $D$ be a $p$-bridge and $D \cap E_{p-1}=\left\{\left[\bar{x}^{0}\right], \ldots,\left[\bar{x}^{n}\right]\right\}$. Then $L_{p}\left[\bar{x}^{0}\right]=0$, $L_{p-1}\left[\bar{x}^{0}\right]=K$, and $\left[\bar{x}^{0}\right]$ is the center of a $(p-1)$-bridge of type $K$. Let $i \in \mathbb{N}$ be the smallest with $L_{p-1}\left[\bar{x}^{i}\right]=0$, and let $j<n$ be the largest with $L_{p-1}\left[\bar{x}^{j}\right]=0$. Let $A_{D}^{1}$ be the arc between $\left[\bar{x}^{0}\right]$ and $\left[\bar{x}^{i}\right]$, and $A_{D}^{2}$ the arc between $\left[\bar{x}^{j}\right]$ and $\left[\bar{x}^{n}\right]$. Then $s T\left(A_{D}^{1}\right)=s T\left(A_{D}^{2}\right)=K$. The arc $A_{D}^{1}$ will be called the first $(p-1)$-semibridge of the $p$-bridge $D$, and $A_{D}^{2}$ the last $(p-1)$-semibridge of $D$. Between $\left[\bar{x}^{i}\right]$ and $\left[\bar{x}^{j}\right]$ there is one or more $(p-1)$-bridges. The ordered set of the first and the last $(p-1)$-semibridges and all $(p-1)$-bridges contained in the $p$-bridge $B$ is called the structure of $B$, denoted by $S(B)$. Note that in the periodic case the first and last $(p-1)$-semibridges are symmetric, but in the strictly preperiodic case they are not. Let $p, q \in \mathbb{Z}_{+}$. Let $B$ be a $p$-bridge of $C$ and let $D$ be a $q$-bridge of $C$. Let $S(B)=\left(A_{B}^{1}, B_{1}, \ldots, B_{n}, A_{B}^{2}\right)$ and $S(D)=\left(A_{D}^{1}, D_{1}, \ldots, D_{m}, A_{D}^{2}\right)$. Then $T(B)=T(D)$ if and only if $m=n$ and $T\left(B_{i}\right)=T\left(D_{i}\right)$ for every $1 \leq i \leq n$.

Lemma 3.21. Let $p \in \mathbb{Z}_{+}$. Let $B \subset C$ be a p-bridge, $B \cap E_{p}=\left\{\left[\bar{x}^{0}\right], \ldots\right.$ $\left.\ldots,\left[\bar{x}^{n}\right]\right\}$ and $S(B)=\left(A_{B}^{1}, B_{1}, \ldots, B_{m}, A_{B}^{2}\right)$. Let $A$ be the arc between $\left[\bar{x}^{0}\right]$ and $\left[\bar{x}^{1}\right]$. Then $\left\{\left[\vec{x}_{-p K}\right]:[\bar{x}] \in A\right\}=\left\{\left[\vec{x}_{-p K}\right]:[\bar{x}] \in B\right\}$ and $A_{B}^{1} \subset A$.

Proof. Suppose that $\left\{\left[\vec{x}_{-p K}\right]:[\bar{x}] \in A\right\} \neq\left\{\left[\vec{x}_{-p K}\right]:[\bar{x}] \in B\right\}$. Let $m=\min \left\{i \in \mathbb{N}: i<n,\left[\vec{x}_{-p K}^{1}\right] \prec\left[\vec{x}_{-p K}^{i}\right]\right\}$. Let $j \in \mathbb{N}, j<m$, be such that $L_{p}\left[\bar{x}^{j}\right] \geq L_{p}\left[\bar{x}^{i}\right]$ for every $i \in \mathbb{N}, i<m$. The point $\left[\bar{x}^{j}\right]$ is closer to $\left[\bar{x}^{m}\right]$ than to $\left[\bar{x}^{0}\right]$. Hence, for the point $\left[\bar{x}^{2 j-m}\right]$ one has $\left[\vec{x}_{-p K}^{1}\right] \prec\left[\vec{x}_{-p K}^{2 j-m}\right]$, contrary to the choice of $\left[\bar{x}^{m}\right]$.

Suppose that $[\vec{c}] \notin \operatorname{int}\left(\sigma^{K}\left\{\left[\vec{x}_{-p K}\right]:[\bar{x}] \in A\right\}\right)$. Then $[\vec{c}] \notin \operatorname{int}\left(\sigma^{K}\left\{\left[\vec{x}_{-p K}\right]\right.\right.$ : $[\bar{x}] \in B\})=\operatorname{int}\left\{\left[\vec{x}_{-(p-1) K}\right]:[\bar{x}] \in B\right\}$. Therefore, $B$ is a $(p-1)$-bridge. Let $B \cap E_{p-1}=\left\{\left[\bar{y}^{0}\right], \ldots,\left[\bar{y}^{l}\right]\right\}$ and let $A_{1}$ be the arc between $\left[\bar{y}^{0}\right]$ and $\left[\bar{y}^{1}\right]$. Then $\left\{\left[\vec{x}_{-p K}\right]:[\bar{x}] \in A\right\}=\left\{\left[\vec{x}_{-(p-1) K}\right]:[\bar{x}] \in A_{1}\right\}$, and thus, $\left\{\left[\vec{x}_{-p K}\right]:[\bar{x}] \in A\right\}=\sigma^{K}\left\{\left[\vec{x}_{-p K}\right]:[\bar{x}] \in A\right\}$. Since $\left\{\left[\vec{x}_{-p K}\right]:[\bar{x}] \in A\right\} \neq I$, this contradicts the fact that $f_{s}$ is l.e.o.

Let $p \in \mathbb{Z}_{+}$and $E_{p}=\left\{\ldots,\left[\bar{x}^{-1}\right],\left[\bar{x}^{0}\right],\left[\bar{x}^{1}\right], \ldots\right\}$ with $\left[\bar{x}^{0}\right]=\bar{c}^{K}$. Let $i \in \mathbb{N}$ be the smallest with $L_{p}\left[\bar{x}^{i}\right]=0$, and let $j \in \mathbb{N}$ be the smallest with $L_{p}\left[\bar{x}^{-j}\right]=0$. Let $F_{p}^{+}$be the arc between $\bar{c}^{K}$ and $\left[\bar{x}^{i}\right]$, and $F_{p}^{-}$the arc between $\left[\bar{x}^{-j}\right]$ and $\bar{c}^{K}$. Then either $s T\left(F_{p}^{+}\right)=K$, or $s T\left(F_{p}^{-}\right)=K$. Without loss of generality we can assume that $s T\left(F_{p}^{+}\right)=K$. The arc $F_{p}^{+}$ will be called the first $p$-semibridge of the composant $C$. Note that if $k \in \mathbb{Z}_{+}$ and $[\bar{x}] \in E_{p}$ are such that $L_{p}[\bar{x}]=k N$, then for every $i \in\{0, \ldots, k-1\}$ and for any two $(p+i)$-semibridges $B_{i}$ and $D_{i}$ which contain $[\bar{x}]$, one has $s T\left(B_{i}\right)=s T\left(D_{i}\right)=K$. For two $(p+k)$-bridges $B_{k}$ and $D_{k}$ which contain $[\bar{x}]$ one has $T\left(B_{k}\right) \neq T\left(D_{k}\right)$, i.e. the composant $C$ does not contain two consecutive $p$-bridges of the same type. 
Finally, we are interested in the folding patterns of the composants without folding point of the continuum $C_{s}$. Denote by $C^{\prime}$ any composant of $C_{s}$ without folding point. Let us sort the $i$-points of $C^{\prime}$ analogously to the way we have sorted the $i$-points of $C$ : For every $p \in \mathbb{Z}_{+}$, the $i$-point $\left[\bar{x}^{\prime}\right] \in C^{\prime}$ is called a p-point if there is $m \in \mathbb{Z}_{+}$with $\left[\vec{x}_{-p K-m}^{\prime}\right]=[\vec{c}]$. The $p$-point $\left[\bar{x}^{\prime}\right]$ has $p$-level $L_{p}\left[\bar{x}^{\prime}\right]=m$ if $\left|x_{-p K-m}^{\prime}-x_{-p K-m}^{\prime *}\right|=1$. For any $p, m \in \mathbb{Z}_{+}$the set

$$
E_{p, m}^{\prime}=\left\{\left[\bar{x}^{\prime}\right] \in C^{\prime}:\left|x_{-p K-m}^{\prime}-x_{-p K-m}^{*}\right|=1\right\}
$$

is the set of all $p$-points of level $m$, and $E_{p}^{\prime}=\bigcup_{m=0}^{\infty} E_{p, m}^{\prime}$ is the set of all $p$-points of the composant $C^{\prime}$. Note that $E_{p+1}^{\prime} \subset E_{p}^{\prime}$ for every $p \in \mathbb{Z}_{+}$. We define, analogously to the case of $C, p$-bridges of the composant $C^{\prime}$, $p$-semibridges of $C^{\prime}$ and their folding patterns.

For an arbitrarily large $k \in \mathbb{N}$, we can find a $p$-point $\left[\bar{x}^{\prime}\right] \in E_{p}^{\prime}$ such that $L_{p}\left[\bar{x}^{\prime}\right]=k N$. For this point there is an order-preserving bijection from $(\mathbb{Z}, \leq)$ to $\left(E_{p}^{\prime}, \preceq\right)$ such that $0 \in \mathbb{Z}$ is mapped to $\left[\bar{x}^{\prime}\right] \in E_{p}^{\prime}$. The points from $E_{p}^{\prime}$ are indexed by $\mathbb{Z}$ and $\left[\bar{x}^{\prime 0}\right]=\left[\bar{x}^{\prime}\right]$. For the point $\left[\bar{x}^{\prime 0}\right]$ and for the $(p+k-1)$ semibridges $A_{1}^{\prime}$ and $A_{2}^{\prime}$ which contain $\left[\bar{x}^{\prime 0}\right]$ one has $T\left(A_{1}^{\prime}\right)=T\left(A_{2}^{\prime}\right)=K$. Hence, the $q$-folding pattern of $A_{1}^{\prime}$ (and of $A_{2}^{\prime}$ ) is the same as the $q$-folding pattern of the first $q$-semibridge $F_{q}$ of the composant $C$, for every $q<p+k$.

On the other hand, let $B^{\prime}$ be a $p$-bridge of the composant $C^{\prime}$ which contains $\left[\bar{x}^{\prime}\right]$. For every $q \leq p$, the $q$-folding pattern of $B^{\prime}$ is determined by $L_{p}\left[\bar{\chi}^{B^{\prime}}\right]$ and is equal to the $q$-folding pattern of some $p$-bridge $D$ of the composant $C$ with $L_{p}\left[\bar{\chi}^{D}\right]=L_{p}\left[\bar{\chi}^{B^{\prime}}\right]$. Note that $B^{\prime}$ is contained in the $(p+i)$-bridge $B_{i}^{\prime}$ for every $i \in \mathbb{N}$. Since $q<p+i$, we can extend the $q$-folding pattern of $B^{\prime}$ to the $q$-folding pattern of $B_{i}^{\prime}$, which is determined by $L_{p}\left[\bar{\chi}^{B_{i}^{\prime}}\right]$, and we can inductively build the folding pattern to the left and to the right of the point $\left[\bar{x}^{\prime}\right]$.

Acknowledgements. I would like to thank Sibe Mardešić for detailed comments on earlier versions of this work.

\section{References}

[B] C. Bandt, Composants of the horseshoe, Fund. Math. 144 (1994), 231-241.

[Ba-Br-D] M. Barge, K. M. Brucks and B. Diamond, Self-similarity in inverse limit spaces of the tent family, Proc. Amer. Math. Soc. 124 (1996), 3563-3570.

[Ba-D] M. Barge and B. Diamond, Homeomorphisms of inverse limit spaces of onedimensional maps, Fund. Math. 146 (1995), 171-187.

[Ba-I] M. Barge and W. T. Ingram, Inverse limits on $[0,1]$ using logistic bonding maps, Topology Appl. 72 (1996), 159-172.

[Ba-M] M. Barge and J. Martin, Endpoints of inverse limit spaces and dynamics, in: Continua. With the Houston Problem Book, H. Cook et al. (eds.), Lecture Notes in Pure and Appl. Math. 170, Dekker, New York, 1995, 165-182. 
[Br-D] K. M. Brucks and B. Diamond, A symbolic representation of inverse limit spaces for a class of unimodal maps, In: Continua. With the Houston Problem Book, H. Cook et al. (eds.), Lecture Notes in Pure and Appl. Math. 170, Dekker, New York, 1995, 207-226.

[Brn1] H. Bruin, Planar embeddings of inverse limit spaces of unimodal maps, Topology Appl. 96 (1999), 191-208.

[Brn2] - Inverse limit spaces of post-critically finite tent maps, Fund. Math. 165 (2000), 125-138.

[Brn3] - Asymptotic arc-components of unimodal inverse limit spaces, Topology Appl. 152 (2005), 182-200.

[C-E] P. Collet and J.-P. Eckmann, Iterated Maps on the Interval as Dynamical Systems, Progr. Phys. 1, Birkhäuser, Basel, 1980.

[I] W. T. Ingram, Periodicity and indecomposability, Proc. Amer. Math. Soc. 123 (1995), 1907-1916.

[K1] L. Kailhofer, A partial classification of inverse limit spaces of tent maps with periodic critical points, Topology Appl. 123 (2002), 235-265.

[K2] - A classification of inverse limit spaces of tent maps with periodic critical points, Fund. Math. 177 (2003), 95-120.

[M-S] W. de Melo and S. van Strien, One-Dimensional Dynamics, Springer, Berlin, 1993.

[P-Y] M. Pollicott and M. Yuri, Dynamical Systems and Ergodic Theory, Cambridge Univ. Press, Cambridge, 1998.

[Š] S. Štimac, Topological classification of Knaster continua with finitely many endpoints, Ph.D. thesis, Univ. of Zagreb, Zagreb, 2002.

Graduate School of Economics and Business

University of Zagreb

Kennedyev $\operatorname{trg} 6$

10000 Zagreb, Croatia

E-mail: sonja@math.hr

Received 30 September 2004;

in revised form 27 February 2006 